\title{
FACTOR SETS AND DIFFERENTIALS ON ABELIAN VARIETIES
}

\author{
BY \\ IACOPO BARSOTTI( ${ }^{(1)}$
}

The main results of this paper are stated in 2.8 and 3.7 ; the notations and language are those used by the author in his previous work; numbers in brackets refer to the bibliography at the end of the paper. Although a few results of $\S \S 1$ and 2 are valid for any characteristic, this paper intends to treat only the case of characteristic zero. The case of positive characteristic is treated in a forthcoming paper, since it needs an analysis of the derivations of higher order, and is connected to the rather surprising fact that an abelian variety over a field of positive characteristic may very well possess exact differentials of the first kind. $\S \S 1$ and 2 contain the proofs of those properties of the differentials of the second kind which are needed in $\$ 3$; these properties are familiar in classical algebraic geometry, but their algebraic proofs are new. While our main interest, in $\S \S 1$ and 2, rests with the differentials of the second kind, certain properties of the differentials of the first kind are also found, usually as special cases; the algebraic proofs of most of these properties are not new, and can be found also in [S. Koizumi, On the differential forms of the first kind on algebraic varieties, J. Math. Soc. Japan vol. 1 (1949) p. 273] and [S. Nakano, On invariant differential forms on group varieties, ibid. vol. 2 (1951) p. 216].

1. The differentials of the first and second kind. Let $V$ be an irreducible variety over the field $k$; let $W$ be an irreducible subvariety of $V$, and set $Q=Q(W / V), \mathfrak{B}=\mathfrak{B}(W / V)$; let $D$ be a derivation on $V$ (see $\S 5$ of $[4])$. We shall say that $D$ is regular at $W$ if $D x \in Q$ when $x \in Q$, and $D x \in \mathfrak{P}$ when $x \in \mathfrak{P}$. If, in addition, $D x \in \mathfrak{P}$ when $x \in Q$, we shall say that $D$ has a zero at $W$. Let $\pi$ be the homomorphic mapping of $Q$ into $k(W)$ whose kernel is $\mathfrak{B}$; if $D$ is regular at $W$, for $x \in Q$ we see that $\pi(D x)$ depends only on $\pi x$, and from this follows the existence of a derivation $\pi D$ on $W$ such that $(\pi D)(\pi x)=\pi(D x)$ for $x \in Q ; \pi D$ is called the derivation induced by $D$ on $W$; clearly, $\pi D=0$ if and only if $D$ has a zero at $W$.

1.1. ThEOREM. Let $V$ be an $n$-dimensional irreducible variety of inseparability 1 over the field $k$; let $W$ be an m-dimensional irreducible subvariety of $V$, simple on $V$, of inseparability 1. Set $Q=Q(W / V), \mathfrak{P}=\mathfrak{B}(W / V)$, and let $\pi$ be the homomorphic mapping of $Q$ onto $k(W)$ whose kernel is $\mathfrak{B}$. Let $x_{1}, \cdots, x_{m}$

Received by the editors April 3, 1956.

(1) (Added March 25, 1956). This paper was submitted to the Amer. J. Math. on July 6, 1955 (received by the editor on July 12), but was not printed in that journal, due to a disagreement on editorial policy. 
be elements of $Q$ such that $k\left(x_{1}, \cdots, x_{m}\right) \subseteq Q$, and that $k(W)$ be an algebraic separable extension of $k\left(\pi x_{1}, \cdots, \pi x_{m}\right)$; let $\left\{x_{m+1}, \cdots, x_{n}\right\}$ be a regular set of parameters of $Q$. There exist derivations $D_{1}, \cdots, D_{n}$ on $V$ such that $D_{i} x_{j}=\delta_{i j}$ (Kronecker's symbol) for $i, j=1, \cdots, n$; these form an independent $k(V)$-basis for the derivations on $V$. The derivations on $V$ regular at $W$ are all and only those of the form $\sum_{i=1}^{n} a_{i} D_{i}$, with $a_{i} \in Q$ for $i \leqq m$, and $a_{i} \in \mathfrak{B}$ for $i>m$; among these, those with a zero at $W$ are all and only those for which $a_{i} \in \mathfrak{P}$ for $i \leqq m$. The derivations $\pi D_{i}(i=1, \cdots, m)$ form an independent $k(W)$-basis for the derivations on $W$.

Proof. Let $F$ be the projective space over $k$ whose n.h.g.p. (nonhomogeneous general point) is $\left\{x_{1}, \cdots, x_{n}\right\}$; let $\left\{y_{1}, \cdots, y_{r}\right\}$ be a n.h.g.p. of $V$, for which $W$ is at finite distance, and let $y_{0}$ be an element of $Q$. Let $V^{\prime}$ be the model of $k(V)$ whose n.h.g.p. is $\left\{y_{0}, \cdots, y_{r}\right\}$; then $W$ corresponds on $V^{\prime}$ to an irreducible variety $W^{\prime}$ such that $Q\left(W^{\prime} / V^{\prime}\right)=Q$. Let $\phi$ be the rational mapping of $V^{\prime}$ onto $F$ generated by the embedding of $k(F)$ into $k\left(V^{\prime}\right)$; let $\left\{Y_{0}, \cdots, Y_{r}\right\}$ be the n.h.g.p. of the ambient space of $\phi[F]$, and let $\left\{f_{1}(Y, x)\right.$, $\left.f_{2}(Y, x), \cdots\right\}$ be a basis of the ideal related to $\phi$ in $k[Y, x]$. The Corollary to Theorem 5.6 of [2] (modified to apply to nonhomogeneous co-ordinates) implies then that the jacobian $J(f(Y, x) ; Y)$ acquires the rank $r+1$ when $\{Y\}$ is replaced by $\{\pi y\}$ and $\{x\}$ by $\{\pi x\}$, hence also when $\{Y\}$ is replaced by $\{y\}$ and $\{x\}$ by $\{x\}$; this, in particular, indicates that ins $(k(V): k(F))=1$, so that $D_{1}, \cdots, D_{n}$ exist and form an independent $k(V)$-basis for the derivations on $V$. From $f_{i}(y, x)=0$ follows $D_{j} f_{i}(y, x)=0$, or

$$
\left[\partial f_{i}(Y, x) / \partial x_{j}\right]_{Y=y}+\sum_{h}\left[\partial f_{i}(Y, x) / \partial Y_{h}\right]_{Y=y} D_{j} y_{h}=0 .
$$

If these are considered as equations in the unknowns $D_{j} y_{0}, \cdots, D_{j} y_{r}$, the previous remark on the rank of $J(f ; Y)$ shows that $D_{j} y_{h} \in Q$, in particular $D_{j} y_{0} \in Q$, valid for any $y_{0} \in Q$. If $y_{0} \in \mathfrak{B}$, then $y_{0}=\sum_{i=m+1}^{n} a_{i} x_{i}, a_{i} \in Q$, so that, for $j \leqq m, D_{j} y_{0}=\sum_{i=m+1}^{n} x_{i} D_{j} a_{i} \in \mathfrak{B}$; hence $D_{1}, \cdots, D_{m}$ are regular at $W$. Moreover, $b D_{j}(j=1, \cdots, n)$ certainly has a zero at $W$ if $b \in \mathfrak{B}$.

Now, let $\sum_{i=1}^{n} a_{i} D_{i}$ be regular at $W$; then $\sum_{i} a_{i} D_{i} x_{j}=a_{j}$ must be in $Q$ if $j \leqq m$, and in $\mathfrak{P}$ if $j>m$; finally, if $\sum_{i} a_{i} D_{i}$ also has a zero at $W$, it must necessarily be $a_{i} \in \mathfrak{P}$ for $i=1, \cdots, n$, as announced. The derivations $\pi D_{i}$ $(i=1, \cdots, m)$ exist and are independent, since $\left(\pi D_{i}\right)\left(\pi x_{j}\right)=\delta_{i j}$, Q.E.D.

The derivations $D_{1}, \cdots, D_{n}$ of 1.1 , in this order, are said to form a canonical $W$-basis for the derivations on $V$, related to the set $\left\{x_{1}, \cdots, x_{n}\right\}$; this set, in turn, is called a set of uniformizing parameters at $W$ on $V$.

Let $V$ be an irreducible variety over $k$; a differential of $k(V)$ over $k$, or on $V$, is a linear mapping $\omega: D \rightarrow \omega D$ of the $k(V)$-module of the derivations on $V$ into $k(V)$; linear in the sense that if $D, D^{\prime}$ are derivations, and $x \in k(V)$, then $\omega D \in k(V), \omega\left(D+D^{\prime}\right)=\omega D+\omega D^{\prime}, \omega(x D)=x \omega D$. If $\omega, \omega^{\prime}$ are differentials on $V$, and $x \in k(V)$, the differentials $\omega+\omega^{\prime}, x \omega=\omega x$ are defined by $\left(\omega+\omega^{\prime}\right) D$ 
$=\omega D+\omega^{\prime} D,(x \omega) D=x(\omega D)$ for any derivation $D$ on $V$. The differentials on $V$ thus form a $k(V)$-module (dual to the $k(V)$-module of the derivations on $V)$. If ins $V=1$, such module has order $n=\operatorname{dim} V$. If $x \in k(V), d x$ (or, if necessary, $\left.d_{V} x\right)$ shall denote the differential on $V$ such that $(d x) D=D x$ for any derivation $D$ on $V$. A differential of the type $d x$ is called an exact differential.

Let $W$ be an irreducible subvariety of $V$; set $Q=Q(W / V), \mathfrak{B}=\mathfrak{B}(W / V)$, and let $\omega$ be a differential on $V$. We say that $\omega$ has a pole at $W$ if either $\omega D \notin Q$ for some derivation $D$ on $V$, regular at $W$, or $\omega D \notin \Re$ for some derivation $D$ on $V$ which has a zero at $W$; if $\omega$ does not have a pole at $W$, it is said to be regular at $W$. If this is the case, and if $\pi$ denotes the homomorphic mapping of $Q$ onto $k(W)$ whose kernel is $\mathfrak{B}$, then, for any derivation $D$ on $V$, regular at $W, \pi(\omega D)$ depends only on $\pi D$, and therefore there exists at least one differential $\omega^{\prime}$ on $W$ such that $\omega^{\prime}(\pi D)=\pi(\omega D)$ for any such $D$. If the $\pi D$ are all the derivations on $W$, as it is under the assumptions of $1.1, \omega^{\prime}$ is unique, and is then denoted by $\pi \omega$ or $\omega \cap W$ or $W \cap \omega$, and called the differential on $W$ induced by $\omega ; \omega$ induces 0 on $W$, or has a zero at $W$, if $\pi \omega=0$, or if $\omega D \in \mathfrak{B}$ for each derivation $D$ on $V$, regular at $W$.

1.2. Theorem. Let $k, V, W,\{x\}, Q, \mathfrak{B}, \pi$ be as in 1.1 ; then the differentials on $V$ regular at $W$ are all and only those of the form $\sum_{i=1}^{n} a_{i} d x_{i}$, with $a_{i} \in Q$ $(i=1, \cdots, n)$; and among these, those with a zero at $W$ are all and only those for which $a_{i} \in \mathfrak{P}(i=1, \cdots, m)$. The differentials $W \cap d x_{i}(i=1, \cdots, m)$ form an independent $k(W)$-basis for the differentials on $W$. If $x \in Q, d x$ is regular at $W$, and $d \pi x=W \cap d x$; if $x \in \mathfrak{B}, d x$ has a zero at $W$.

Proof. For $i=1, \cdots, n, d x_{i}$ is regular at $W$; in fact, if $D_{1}, \cdots, D_{n}$ have the same meaning as in 1.1 , and if $D=\sum_{i} a_{i} D_{i}$ is regular at $W$, we have $\left(d x_{i}\right) D=D x_{i}=a_{i}$, and this, by 1.1 , belongs to $Q$, and also to $\mathfrak{B}$ if $D$ has a zero at $W$. If $\omega=\sum_{i} b_{i} d x_{i}$ is regular at $W$, then $\omega D=\sum_{i} b_{i} a_{i}$ must belong to $Q$ if $a_{1}, \cdots, a_{m} \in Q$ and $a_{m+1}, \cdots, a_{n} \in \mathfrak{P}$, and to $\mathfrak{B}$ if also $a_{1}, \cdots, a_{m} \in \mathfrak{P}$. This is true if and only if $b_{1}, \cdots, b_{n} \in Q$; and $\omega$ has a zero at $W$ if and only if $\sum_{i} b_{i} a_{i} \in \mathfrak{B}$ for $a_{1}, \cdots, a_{m} \in Q, a_{m+1}, \cdots, a_{n} \in \mathfrak{B}$; this is true if and only if $b_{1}, \cdots, b_{m} \in \mathfrak{B}$ and $b_{m+1}, \cdots, b_{n} \in Q$. The differentials $W \cap d x_{i}(i=1, \cdots, m)$ exist, and are independent over $k(W)$; hence they form a $k(W)$-basis for the differentials on $W$.

If $x \in Q$, then $d x=\sum_{i}\left(D_{i} x\right) d x_{i}$, and this is regular at $W$ because $D_{i} x \in Q$ for $i=1, \cdots, n$; if $x \in \mathfrak{B}$, then $x=\sum_{j=m+1}^{n} y_{j} x_{j}$, with $y_{j} \in Q$; hence $d x$ $=\sum_{j=m+1}^{n} y_{j} d x_{j}+x_{j} d y_{j}$; since each $d x_{j}$ and each $x_{j} d y_{j}$ has a zero at $W$, the same is true of $d x$. The statement $W \cap d x=d \pi x$ is obvious, Q.E.D.

The differentials $d x_{1}, \cdots, d x_{n}$ of 1.2 , ordered so that those with a zero at $W$ are the last $n-m$, are said to form a canonical $W$-basis for the differentials on $V$, related to the set $\left\{x_{1}, \cdots, x_{n}\right\}$.

1.3. Lemma. Let $V, W$ be as in 1.1 , and let $\omega$ be a differential on $V$ which 
has a pole at $W$; then $\omega$ has a pole at some $(n-1)$-dimensional irreducible subvariety of $V$ containing $W$.

Proof. Set $Q=Q(W / V), \mathfrak{B}=\mathfrak{B}(W / V)$. If $\omega$ has a pole at $W$, then either $\omega D \notin Q$ for some derivation $D$ on $V$, regular at $W$, or $\omega D \notin \mathfrak{B}$ for some derivation $D$ on $V$ with a zero at $W$. In the first case, set $\omega D=q p^{-1}$, where $q \in Q$, $p \in \mathfrak{B}$, and $p, q$ have no common factors (here one should remember that every minimal prime ideal of $Q$ is principal; see [8]). If $D^{\prime}=p D, D^{\prime}$ has a zero at $W$; let $U$ be the center on $V$ of a prime divisor $v$ of $V$ such that $Q \subseteq R_{v}$ and that $v(p)>0$; then $D^{\prime} x \in p Q \subseteq \mathfrak{B}_{v}$ for $x \in Q$, so that if $a=x y^{-1} \in R_{v}$, with $x \in Q, y \in Q-p Q$, we have $D^{\prime} a=y^{-1} D^{\prime} x-x y^{-2} D^{\prime} y \in \mathfrak{B}_{v}$. Hence $D^{\prime}$ has a zero at $U$; but $\omega D^{\prime}=p \omega D=q \notin \mathfrak{P}_{v}$, which proves that $\omega$ has a pole at $U$. In the second case, i.e. when $\omega D \notin \mathfrak{B}$ and $D$ has a zero at $W$, we may assume $\omega D=q$ to be a unit of $Q$, otherwise this case would fall within the first case. If $\left\{D_{1}, \cdots, D_{n}\right\}$ is a canonical $W$-basis for the derivations on $V$, we have $D=\sum_{i} a_{i} D_{i}, a_{i} \in \mathfrak{B}$, and $q=\omega D=\sum_{i} a_{i} \omega D_{i}$. Unless $\omega$ is such that the first case applies, we must have $\omega D_{i} \in Q$ for $i \leqq m$, and therefore $\sum_{i=m+1}^{n} a_{i} \omega D_{i}$ is a unit of $Q$, while each $a_{i} \omega D_{i}$ belongs to $Q$. As a consequence, $a_{i} \omega D_{i}$ is a unit of $Q$ for some $i>m$, say $i=n$. Now, let $U, v$ be related to $a_{n}$ as they were before to $p$; if $D^{\prime}=a_{n} D_{n}$, we have $D^{\prime} x \in a_{n} Q \subseteq \mathfrak{P}_{v}$ for $x \in Q$, and therefore also for $x \in R_{v}$; hence $D^{\prime}$ has a zero at $U$, but $\omega D^{\prime} \notin \Re_{v}$. This proves that $\omega$ has a pole at $U$, Q.E.D.

The sentence " $U$ is a pole of $\omega$ " shall mean, from now on, that $\omega$ has a pole at $U$, and that $U$ has dimension $n-1$, if $n$ is the dimension of $V$.

1.4. TheOREM. Let $V$ be an $n$-dimensional irreducible variety over $k$; let $W$ be an irreducible proper subvariety of $V$, simple on $V$, and of inseparability 1; let $\omega$ be a differential on $V$. Then $\omega$ has a pole at $W$ if and only if $W$ is a subvariety of a pole of $\omega$. If $x \in k(V)$, and $V$ has no $(n-1)$-dimensional singularity, and no (n-1)-dimensional irreducible subvariety of inseparability $>1$, each pole of $d x$ is a pole of $x$; under the same assumptions, $\omega$ has finitely many poles. If, in addition, $k$ has characteristic 0 , each pole of $x$ is a pole of $d x$.

Proof. If $\omega$ has a pole at $W$, then a pole of $\omega$ contains $W$ by 1.3. If $W \subseteq U$ and $U$ is a pole of $\omega$, let $\left\{d x_{1}, \cdots, d x_{n}\right\}$ be a canonical $W$-basis for the differentials on $V$, and write $\omega=\sum_{i} a_{i} d x_{i}$; should $\omega$ not have a pole at $W$, we would have $a_{i} \in Q(W / V) \subseteq Q(U / V)$ for each $i$ by 1.2 . Since, by $1.2, d x_{i}$ is regular at $U$, the same would be true of $\omega$, a contradiction. Hence $\omega$ has a pole at $W$. If $U$ is now any $(n-1)$-dimensional irreducible subvariety of $V$, simple on $V$, and if ins $U=1$, from $x \in Q(U / V)$ follows that $d x$ is regular at $U$ by 1.2 ; hence each pole of $d x$, under the additional conditions mentioned in the statement, is a pole of $x$. Therefore the poles of $\omega$ are to be found among the poles of $x_{1}, \cdots, x_{n}, a_{1}, \cdots, a_{n}$, and are finite in number. If $k$ has characteristic 0 , and $U$ is a pole of $x$, we can write $x=u t^{r}$, where $u$ is a unit of $Q(U / V), t$ generates $\mathfrak{B}(U / V)$, and $r<0$. Then $d x=r u t^{r-1} d t+t^{r} d u$; let 
$\left\{t_{1}, \cdots, t_{n}\right\}$ be a set of uniformizing parameters at $U$ on $V$, such that $t_{n}=t$, and let $\left\{D_{1}, \cdots, D_{n}\right\}$ be the canonical $U$-basis for the derivations on $V$ which is related to $\{t\}$. Then $(d x)\left(t D_{n}\right)=r u t^{r}+t^{r+1} D_{n} u$; since $t D_{n} u \in \mathfrak{B}(U / V)$, it follows that $t^{r+1} D_{n} u \in t^{r+1} Q(U / V)$, while $r u t^{r} \notin t^{r+1} Q(U / V)$; hence $(d x)\left(t D_{n}\right)$ $\notin Q(U / V)$, and therefore $U$ is a pole of $d x$, since $t D_{n}$ has a zero at $U$ by 1.1 , Q.E.D.

1.5. Theorem. Let $U, W, V$ be irreducible varieties over $k$, such that (1) $U \subset W \subset V$, (2) $U$ is simple on $W$ and $V$, and (3) ins $U=1$; let $\omega$ be a differential on $V$, regular at $U$. Then $\omega$ is regular at $W$, and $\omega \cap U=(\omega \cap W) \cap U$.

Proof. By conditions (2) and (3), if the dimensions of $W, V$ are $m, n$ respectively, it is possible to find a set of uniformizing parameters $\left\{x_{1}, \cdots, x_{n}\right\}$ at $U$ on $V$ which is also a set of uniformizing parameters at $W$ on $V$, and such that if $\pi$ is the homomorphic mapping of $Q(W / V)$ onto $k(W)$ whose kernel is $\mathfrak{P}(W / V),\left\{\pi x_{1}, \cdots, \pi x_{m}\right\}$ is a set of uniformizing parameters at $U$ on $W$. If $\omega$ is regular at $U$, we have $\omega=\sum_{i=1}^{n} a_{i} d x_{i}$, with $a_{i} \in Q(U / V)$, by 1.2 ; hence $a_{i} \in Q(W / V)$, and $\omega$ is regular at $W$. But then, by $1.2, \omega \cap W=\sum_{i=1}^{m}\left(\pi a_{i}\right) d \pi x_{i}$; now, $\pi a_{i} \in Q(U / W)$, so that $\omega \cap W$ is regular at $U$ by 1.2 , and the relation $(\omega \cap W) \cap U=\omega \cap U$ follows easily, Q.E.D.

A differential $\omega$ on $V$ is said to be of the first kind (on $V$ ) if it is regular at each irreducible subvariety of $V$; by 1.4 , if $V$ has no singularity, and no irreducible subvariety with inseparability $>1$, a differential on $V$ is of the first kind if and only if it has no poles (of dimension $n-1$ if $n=\operatorname{dim} V$ ). Two differentials $\omega, \omega^{\prime}$ on $V$ are said to be equivalent, in symbols $\omega \sim \omega^{\prime}$, if $\omega^{\prime}-\omega$ is exact. A differential $\omega$ on $V$ is said to be of the second kind (on $V$ ) if for each irreducible proper subvariety $W$ of $V$ there exists a differential $\omega^{\prime} \sim \omega$ which is regular at $W$. From 1.5 we obtain:

1.6. Corollary. Let $W$ be an irreducible subvariety of the irreducible variety $V$ over the perfect field $k$, and assume each point of $W$ to be simple on $W$ and $V$. Let $\omega$ be a differential on $V$, regular at $W$; if $\omega$ is of the first (second) kind, so is $W \cap \omega$.

Two derivations $D, \Delta$ on $V$ are said to commute if $D \Delta x=\Delta D x$ for each $x \in k(V)$; a differential $\omega$ on $V$ is said to be closed if $D(\omega \Delta)=\Delta(\omega D)$ for each pair $\{D, \Delta\}$ of derivations on $V$ which commute. Any exact differential on $V$ is closed; and any differential on $V$ is closed if $\operatorname{dim} V=1$, as will clearly appear from the following result:

1.7. Lemma. Let $V$ be an n-dimensional irreducible variety over $k$, of inseparability 1. A differential $\omega$ on $V$ is closed if and only if $D_{i}\left(\omega D_{j}\right)=D_{j}\left(\omega D_{i}\right)$ for $i, j=1, \cdots, n$, where $\left\{D_{1}, \cdots, D_{n}\right\}$ is a $k(V)$-basis for the derivations on $V$, consisting of mutually commutative derivations.

Proof. If $D=\sum_{i} a_{i} D_{i}$, and $\Delta=\sum_{i} b_{i} D_{i}$ are commutative derivations on 
$V$, with $a_{i}, b_{i} \in k(V)$, we have $D b_{i}=\Delta a_{i}$, and $D(\omega \Delta)=\sum_{i}\left(D b_{i}\right) \omega D_{i}+b_{i} D\left(\omega D_{i}\right)$ $=\sum_{i}\left(\Delta a_{i}\right) \omega D_{i}+a_{i} \Delta\left(\omega D_{i}\right)=\Delta(\omega D)$, Q.E.D.

It is evident that if $\omega$ is closed, also $W \cap \omega$ is closed if it exists.

Let $V$ be an absolutely irreducible variety over $k$, and let $K$ be an extension of $k$. If $D$ is a derivation on $V$, there is a unique derivation $D_{K}$ on $V_{K}$ such that $D_{K} x=D x$ for any $x \in k(V) \subseteq K\left(V_{K}\right)$. Likewise, if $\omega$ is a differential on $V$, there is a unique differential $\omega_{K}$ on $V_{K}$ such that $\omega_{K} D_{K}=\omega D$ for any derivation $D$ on $V$; in particular, $d_{V_{K}} x=\left(d_{V} x\right)_{K}$ for $x \in k(V)$. A somewhat different situation arises in considering two irreducible varieties $V, V^{\prime}$ such that $k(V) \subseteq k\left(V^{\prime}\right)$ and ins $V=$ ins $\left(k\left(V^{\prime}\right): k(V)\right)=1$; in this case, for $x \in k(V)$, we have $d_{V} x=0$ if and only if $d_{V} x=0$; if $\omega$ is a differential on $V$, and $\omega=\sum_{i} a_{i} d_{V} x_{i}$, where $a_{i}, x_{i} \in k(V)$, then $\sum_{i} a_{i} d_{V^{\prime}} x_{i}$ gives a differential on $V^{\prime}$, which does not depend on the choice of the $x$ 's, and which is 0 if and only if $\omega=0$. Such differential is called the extension of $\omega$ on $V^{\prime}$. A particular case arises when $V^{\prime}=V \times W$, where $W$ is another absolutely irreducible variety over $k$ ( $V$ being also absolutely irreducible in this case); then the extension of $\omega$ on $V^{\prime}$ will be denoted by $\omega \times W$ or $W \times \omega$; there is an obvious one-to-one correspondence $\omega \times W \rightarrow \omega_{K}$, for $K=k(W)$. Also, $\omega \times W$ or $\omega_{K}$ are closed if and only if $\omega$ is closed.

1.8. Lemma. Let $V$ be an irreducible variety without singularities over the algebraically closed field $k$; let $K$ be an extension of $k$, and let $\omega$ be a differential on $V$; then $\omega_{K}$ is of the first (second) kind if and only if $\omega$ is of the first (second) kind.

Proof. Set $n=\operatorname{dim} V, d=d_{V}, d^{\prime}=d_{V_{K}}$; assume $\omega$ to be of the second kind' and let $U$ be a nonempty irreducible subvariety of $V_{K}$; there exists a point $P \in V$ such that $Q(P / V) \subseteq Q\left(U / V_{K}\right)$, and there exists an $f \in k(V)$ such that $\omega-d f$ is regular at $P$; in particular, $f=0$ if $\omega$ is of the first kind. Thus, $\omega-\bar{d} f$ $=\sum_{i} a_{i} d x_{i}$, where $a_{i}, x_{i} \in Q(P / V)$, by 1.2 ; but then, again by $1.2, \omega_{K}-d^{\prime} f$ is regular at $U$, so that $\omega_{K}$ is of the second kind, or of the first kind if $f=0$.

Now, assume $\omega_{K}$ to be of the second kind; let $U$ be a nonempty irreducible subvariety of $V$, and let $f \in K\left(V_{K}\right)$ be such that $\omega_{K}-d^{\prime} f$ is regular at $U_{K}$; here, $f=0$ if $\omega_{K}$ is of the first kind. Let $v$ be any place of $K$ over $k$, whose extension $w$ to $K\left(V_{K}\right)$ over $k(V)$ is such that $w(f) \geqq 0$, and denote by - the homomorphic image of an element of $R_{w} \bmod \mathfrak{P}_{w}$. Write $\omega_{K}-d^{\prime} f=\sum_{i} a_{i} d^{\prime} x_{i}$, with $a_{i} \in Q\left(U_{K} / V_{K}\right),\left\{x_{1}, \cdots, x_{n}\right\}$ being a set of uniformizing parameters at $U$ on $V$. The poles of $\omega_{K}-d^{\prime} f$ are among the poles of the $a_{i}$ and the $x_{i}$, by 1.4 , and none of them contains $U_{K}$, again by 1.4 ; hence, after a suitable choice of $v$, none of the poles of any $\bar{a}_{i}$ or any $x_{i}$ contains $U$, and therefore $\omega-d \bar{f}$ is regular at $U$, Q.E.D.

If $V$ is an irreducible variety over the field $k$, we shall denote by $D_{1}(V)$, $D_{2}(V), D_{e}(V)$ respectively the $k$-modules of the closed differentials of the first kind on $V$, of the closed differentials of the second kind on $V$, and of the 
exact differentials on $V$; clearly, $D_{1}(V)$ and $D_{e}(V)$ are sub-modules of $D_{2}(V)$, their intersection being 0 if $k$ has characteristic 0 , by 1.4. If $V$ is absolutely irreducible, we shall say that an independent $k$-basis $\left\{\omega_{1}, \omega_{2}, \cdots\right\}$ of $D_{1}(V)$ is an absolute basis, if $\left\{\left(\omega_{1}\right)_{K},\left(\omega_{2}\right)_{K}, \cdots\right\}$ is a $K$-basis of $D_{1}\left(V_{K}\right)$, for each algebraically closed extension $K$ of $k$. And a set $\left\{\omega_{1}, \omega_{2}, \cdots\right\}$ of elements of $D_{2}(V)$, whose images mod $D_{e}(V)$ form an independent $k$-basis of $\mathscr{D}_{2}(V) / D_{e}(V)$, is said to represent an absolute basis, if the images of $\left(\omega_{1}\right)_{K},\left(\omega_{2}\right)_{K}, \cdots$, $\bmod \mathscr{D}_{e}\left(V_{K}\right)$, form a $K$-basis of $\mathfrak{D}_{2}\left(V_{K}\right) / D_{e}\left(V_{K}\right)$, for any algebraically closed extension $K$ of $k$.

If $\left\{\omega_{1}, \omega_{2}, \cdots\right\}$ is an absolute basis of $D_{1}(V)$, or, respectively, a representative of an absolute basis of $D_{2}(V) / D_{e}(V)$, then $\left\{\left(\omega_{1}\right)_{K},\left(\omega_{2}\right)_{K}, \cdots\right\}$ is a $K$-basis of $D_{1}\left(V_{K}\right)$, or, respectively, represents a $K$-basis of $D_{2}\left(V_{K}\right) / D_{e}\left(V_{K}\right)$, for any extension $K$ of $k$. We shall give the proof of this statement in the second, less obvious, case. Let $K$ be an extension of $k$, and let $\omega \in D_{2}\left(V_{K}\right)$; then, for a suitable finite extension $H$ of $K$, we can find an $x \in H\left(V_{H}\right)$, and elements $a_{1}, a_{2}, \cdots \in H$, such that $\omega_{H}-d_{V_{H}} x=\sum_{i} a_{i}\left(\omega_{i}\right)_{H}$. Let $\left\{b_{1}=1\right.$, $\left.b_{2}, \cdots, b_{r}\right\}$ be an independent $K$-basis of $H$; then $\{b\}$ is also an independent $K\left(V_{K}\right)$-basis of $H\left(V_{H}\right)$. Write $x=\sum_{j} x_{j} b_{j}, x_{j} \in K\left(V_{K}\right)$, and $a_{i}=\sum_{j} a_{i j} b_{j}$, $a_{i j} \in K$. Then $\omega_{H}-d_{V_{H}} x_{1}-\sum_{i} a_{i 1}\left(\omega_{i}\right)_{H}=\sum_{j=2}^{r}\left(d_{V_{H}} x_{j}+\sum_{i} a_{i j}\left(\omega_{i}\right)_{H}\right) b_{j}$. If $D$ is any derivation on $V$, this gives $\omega D_{K}-D_{K} x_{1}-\sum_{i} a_{i 1} \omega_{i} D=\sum_{j=2}^{r}\left(D_{K} x_{j}\right.$ $\left.+\sum_{i} a_{i j} \omega_{i} D\right) b_{j}$; since the left member, and each $D_{K} x_{j}+\sum_{i} a_{i j} \omega_{i} D$, for $j>1$, belong to $K\left(V_{K}\right)$, we conclude that $\omega D_{K}-D_{K} x_{1}=\sum_{i} a_{i 1} \omega_{i} D$, or that $\omega-d_{V_{K}} x_{1}$ $=\sum_{i} a_{i 1}\left(\omega_{i}\right)_{K}$, as claimed.

1.9. THEOREM. Let $V_{1}, V_{2}$ be irreducible varieties without singular points over the algebraically closed field $k$, and set $V=V_{1} \times V_{2}$. For a given $j(=1,2)$, assume $\omega_{i} \in D_{j}\left(V_{i}\right)(i=1,2)$; then $\omega_{1} \times V_{2}+\omega_{2} \times V_{1} \in D_{j}(V)$. If $\omega_{1}, \omega_{2}$ are differentials on $V_{1}, V_{2}$ respectively, and $\omega=\omega_{1} \times V_{2}+\omega_{2} \times V_{1} \in D_{j}(V)(j=1$ or 2$)$, then $\omega_{i} \in D_{j}\left(V_{i}\right)$ for $i=1,2$; also, $\omega_{1}, \omega_{2}$ are uniquely determined by $\omega$. If $\mathfrak{D}_{1}\left(V_{i}\right)$ has an absolute basis for $i=1,2$, and $k$ has characteristic zero, then $\mathfrak{D}_{1}(V)$ has an absolute basis, and each element of $D_{1}(V)$ can be expressed in the form $\omega_{1} \times V_{2}$ $+\omega_{2} \times V_{1}$, with $\omega_{i} \in D_{1}\left(V_{i}\right)$. If there exists a representative of an absolute basis of $\mathfrak{D}_{2}\left(V_{i}\right) / D_{e}\left(V_{i}\right)$ for $i=1,2$, and $k$ has characteristic zero, then there exists a representative of an absolute basis of $D_{2}(V) / D_{e}(V)$, and each element of $D_{2}(V)$ is equivalent $(\sim)$ to an element of the form $\omega_{1} \times V_{2}+\omega_{2} \times V_{1}$, with $\omega_{i} \in D_{2}\left(V_{i}\right)$ $(i=1,2)$, uniquely determined but for equivalence.

Proof. PART 1. Set $n_{i}=\operatorname{dim} V_{i}$; if $\omega=\omega_{1} \times V_{2}+\omega_{2} \times V_{1}$, any pole of $\omega$ is of type $U_{1} \times V_{2}$ or $U_{2} \times V_{1}$, with $U_{i}$ a pole of $\omega_{i}$. Hence, by $1.4, \omega \in D_{i}(V)(i=1,2)$ if and only if $\omega_{j} \in D_{i}\left(V_{j}\right)$ for $j=1,2$.

PART 2. Assume $k$ to have characteristic zero, and let $\left\{\alpha_{i 1}, \alpha_{i 2}, \cdots\right\}$ represent an absolute basis of $\mathscr{D}_{2}\left(V_{i}\right) / D_{e}\left(V_{i}\right)(i=1,2)$. Let $\omega \in \mathscr{D}_{2}(V)$, and write $\omega=\sum_{i} a_{i} d x_{i}+\sum_{j} b_{j} d y_{j}$, where $a_{i}, b_{j} \in k(V)$, and $\left\{x_{1}, \cdots, x_{n_{1}}\right\}$, $\left\{y_{1}, \cdots, y_{n_{2}}\right\}$ are algebraically independent (over $k$ ) elements of $k\left(V_{1}\right)$, 
$k\left(V_{2}\right)$ respectively, such that $k\left(V_{1}\right), k\left(V_{2}\right)$ are finite separable extensions of, respectively, $k(x)$ and $k(y)$. Set $K=k\left(V_{1}\right), H=k\left(V_{2}\right), d^{*}=d_{\left(V_{1}\right)_{H}}, \omega^{*}$ $=\sum_{i} a_{i} d^{*} x_{i}$. Then $\omega^{*}$ is a closed differential on $\left(V_{1}\right)_{H} ;$ if $W$ is an irreducible subvariety of $\left(V_{1}\right)_{H}$ at which $\omega^{*}$ has a pole, set $W^{\prime}=D_{W, V_{2}}$, so that $W^{\prime}$ is a subvariety of $V$ such that $Q\left(W^{\prime} / V\right)=Q\left(W /\left(V_{1}\right)_{H}\right)$. There exists a $z \in k(V)$ such that $\omega-d z$ is regular at $W^{\prime}$; but then $\omega^{*}-d^{*} z$ is regular at $W$. This proves that $\omega^{*} \in D_{2}\left(\left(V_{1}\right)_{H}\right)$; as a consequence, there exists an $x \in k(V)$ such that $\omega^{*}-d^{*} x$ is a linear combination, with coefficients in $H$, of, say, $\left(\alpha_{11}\right)_{H}, \cdots,\left(\alpha_{1 r}\right)_{H}$. Set $\alpha_{18}=\sum_{j} t_{s j} d_{1} x_{j}, t_{s j} \in K$; then there are elements $v_{1}, \cdots, v_{r}$ of $H$ such that $\omega-d x=\sum_{i j} v_{i} t_{i j} d x_{j}+\sum_{j} w_{j} d y_{j}$, with $w_{j} \in k(V)$. This is a closed differential; if $D_{i}, \Delta_{j}$ are the derivations on $V$ such that $D_{i} x_{j}=\delta_{i j}, D_{i} y_{j}=0, \Delta_{i} x_{j}=0, \Delta_{i} y_{j}=\delta_{i j}$, we must have $D_{i} w_{j}=\sum_{h} t_{h i} \Delta_{j} v_{h}$. This implies that the poles of $d^{*} w_{j}$ are all of the type $U_{H}$, with $U$ an $\left(n_{1}-1\right)$ dimensional irreducible subvariety of $V$; consequently, by 1.4 , the poles of $w_{j}$ on $\left(V_{1}\right)_{H}$ are of the same type, and therefore $w_{j} \in K \times H$ (direct product over $k$ ). Write then $w_{j}=\sum_{s} c_{j s} e_{j s}$, with $c_{j s} \in K, e_{j s} \in H$, and $e_{j 1}, e_{j 2}, \cdots$ linearly independent over $k$, hence over $K$. Then $\sum_{h}\left(\Delta_{j} v_{h}\right) t_{h i}=D_{i} w_{j}=\sum_{s} e_{j s} D_{i} c_{j s}$; this shows, by (99) of [1], that there are elements $\beta_{j h_{s}} \in k$ such that $\Delta_{j} v_{h}$ $=\sum_{s} \beta_{h_{s}} e_{j s}$, and that $D_{i} c_{j s}=\sum_{h} \beta_{j h_{s}} t_{h i}$, so that $d_{1} c_{j_{s}}=\sum_{h i} \beta_{j h_{s}} t_{h i} d_{1} x_{i}$ $=\sum_{h} \beta_{j h s} \alpha_{1 h}$. Since $\alpha_{11}, \cdots, \alpha_{1 r}$ are independent over $k \bmod D_{e}\left(V_{1}\right)$, we conclude that $\beta_{j h s}=0$, so that $\Delta_{j} v_{h}=0, v_{h} \in k$; also, $D_{i} c_{j s}=0, c_{j s} \in k$, and $w_{j} \in H$. Thus $\omega-d x=\omega_{1} \times V_{2}+\omega_{2} \times V_{1}$, where $\omega_{1}=\sum_{i} v_{i} \alpha_{i}$ is a differential on $V_{1}$, and $\omega_{2}=\sum_{j} w_{j} d_{2} y_{j}$ is a differential on $V_{2}$. By Part 1 of this proof, $\omega_{1}$ and $\omega_{2}$ are closed and of the second kind; they are unique but for equivalence $(\sim)$, because if $\dot{\omega}=\omega_{1} \times V_{2}+\omega_{2} \times V_{1} \sim 0$, then, for a generic $P_{2} \in V_{2}$, also $\omega_{1} \times P_{2}$ $=\omega \cap\left(V_{1} \times P_{2}\right) \sim 0$.

PART 3. The statement concerning the case in which $\omega$ is of the first kind is proved in the same manner (actually simpler) used in Part 2.

PART 4. The statement concerning the existence of absolute bases is a direct consequence of Parts 3 and 4, Q.E.D.

2. Application to abelian varieties. Let $G$ be a group-variety, without singular points outside the degeneration locus $F$, over the field $k$. If $P$ is a rational point of $G, \sigma_{P}$ and $\tau_{P}$ will have the usual meanings (see [3] and [4]). If $D$ is a derivation on $G$, we shall denote by $\sigma_{P} D$ the derivation on $G$ such that $\left(\sigma_{P} D\right)\left(\sigma_{P} x\right)=\sigma_{P}(D x)$ for any $x \in k(G) ; \tau_{P} D$ is similarly defined. When $k$ is algebraically closed, and according to $\$ 5$ of [4], $D$ is left (respectively right) invariant if and only if $\sigma_{P} D=D$ (respectively $\tau_{P} D=D$ ) for any $P \in G-F$. Let $\omega$ be a differential on $G$; we shall denote by $\sigma_{P} \omega$ the differential on $G$ such that $\left(\sigma_{P} \omega\right)\left(\sigma_{P} D\right)=\sigma_{P}(\omega D)$ for any derivation $D$ on $G$; in particular, $\sigma_{P} d x$ $=d \sigma_{P} x$ for $x \in k(G) ; \tau_{P} \omega$ is similarly defined. If $k$ is algebraically closed, $\omega$ is said to be left (right) invariant if $\sigma_{P} \omega=\omega$ (if $\left.\tau_{P} \omega=\omega\right)$ for any $P \in G-F$; if both relations are fulfilled, $\omega$ is said to be invariant. Under the same conditions, $\omega$ is said to be left (right) semi-invariant if for each $P \in G-F$ there exists an 
$x_{P} \in k(G)$ such that $\sigma_{P} \omega-\omega=d x_{P}$ (respectively $\tau_{P} \omega-\omega=d x_{P}$ ); if both relations are fulfilled (not necessarily for the same $x_{P}$ ), $\omega$ is semi-invariant.

2.1. TheOREM. Let $A$ be an n-dimensional nonsingular abelian variety over the algebraically closed field $k$. Then the invariant differentials on $A$ are all elements of $D_{1}(A)$, and form a k-module of order $n$. Also, each differential of the first kind on $A$ is invariant, hence closed, and $D_{1}(A)$ has an absolute basis.

Proof. Let $D_{1}, \cdots, D_{n}$ be the invariant derivations on $A$ defined in $\$ 5$ of [4], and let $\omega_{i}$ be the differential on $A$ defined by $\omega_{i} D_{j}=\delta_{i j}$. Then $\omega_{1}, \cdots$, $\omega_{n}$ are invariant, and linearly independent over $k$ (also over $k(A)$ ). If $\omega$ is an invariant differential on $A$, set $\omega=\sum_{i=1}^{n} a_{i} \omega_{i}$, with $a_{i} \in k(A)$; for $P \in A$, $\omega=\sigma_{P} \omega=\sum_{i}\left(\sigma_{P} a_{i}\right) \omega_{i}$, or $\sigma_{P} a_{i}=a_{i}$, or $a_{i} \in k$. Hence the invariant differentials on $A$ form a $k$-module of order $n$. If $\omega$ is one of them, and $U$ is a pole of $\omega$, $\sigma_{P} U$ is also a pole of $\omega$ for each $P \in A$. Hence $\omega$ has infinitely many poles, a contradiction to 1.4. Therefore $\omega$ is of the first kind; in order to show that it is closed, it is sufficient to prove that each $\omega_{i}$ is closed; this will be a consequence of 1.7 if we can prove that $D_{i} D_{j}=D_{j} D_{i}(i, j=1, \cdots, n)$.

For sake of brevity, we shall make use of the functional notation of analysis, in spite of its ambiguous meaning. Let $\left\{x_{1}, \cdots, x_{m}\right\}$ be a n.h.g.p. of $A$ such that $x_{i}=0$ at $E_{A}$ (identity), and that $\left\{x_{1}, \cdots, x_{n}\right\}$ form a regular set of parameters of $Q\left(E_{A} / A\right)$. We shall consider an algebraically closed extension $K$ of $k$, of sufficiently high transcendency over $k$, and shall say that a point $P$ of $A_{K}$, at finite distance for $\{x\}$, is general for $A$, if the values $y_{1}, \cdots, y_{m} \in K$ of, respectively, $x_{1}, \cdots, x_{m}$, at $P$ are such that $k(y)$ is isomorphic to $k(x)$ over $k$, in an isomorphism in which $y_{i}$ corresponds to $x_{i}(i=1, \cdots, m)$. There are rational functions $g_{1}, \cdots, g_{m}$ of two sets of indeterminates $\left\{X_{1}, \cdots, X_{m}\right\},\left\{Y_{1}, \cdots, Y_{m}\right\}$, such that, for generic points $P, Q$ of $A$, the co-ordinates of $P Q$ are obtained by replacing, in $g_{1}, \cdots, g_{m}$, the co-ordinates of $P$ for $\{X\}$, and of $Q$ for $\{Y\}$. Then $\$ 5$ of [4] states that $D_{i} x_{j}=\left[\partial g_{j}(x, t) / \partial t_{i}\right]_{t=0}$, if $\{t\}$ are the co-ordinates of a point of $A_{K}$, general for $A$, and such that $k(x, t)$ has transcendency $2 n$ over $k$. The previous formula is valid for $i=1, \cdots, n$ and $j=1, \cdots, m$; for the purpose of computing the partial derivatives, $t_{n+1}, \cdots, t_{n}$ must be considered as functions of $t_{1}, \cdots, t_{n}$. Then

$$
\begin{aligned}
D_{h} D_{i} x_{j} & =D_{h}\left[\partial g_{j}(x, t) / \partial t_{i}\right]_{t=0} \\
& =\sum_{r=1}^{n}\left[\partial^{2} g_{j}(x, t) / \partial t_{i} \partial x_{r}\right]_{t=0} D_{h} x_{r} \\
& =\sum_{r}\left[\partial^{2} g_{j}(x, t) / \partial t_{i} \partial x_{r}\right]_{t=0}\left[\partial g_{r}(x, z) / \partial z_{h}\right]_{z=0} \\
& =\sum_{r}\left[\partial^{2} g_{j}(g(x, z), t) / \partial t_{i} \partial \xi_{r}(x, z)\right]_{t=z=0}\left[\partial g_{r}(x, z) / \partial z_{h}\right]_{z=0} \\
& =\left[\partial^{2} g_{j}(x, g(z, t)) / \partial t_{i} \partial z_{h}\right]_{t=z=0} ;
\end{aligned}
$$


this expression remains unchanged after interchanging $i$ and $h$; therefore $D_{h} D_{i}=D_{i} D_{h}$, as claimed. This proves completely the first statement of the theorem.

Now, let $\omega$ be a differential of the first kind on $A$; let $D$ be any invariant derivation on $A$, and set $a=\omega D$. If $V$ is any $(n-1)$-dimensional irreducible subvariety of $A, Q(V / A)$ is the join of the $Q(P / A)$ for $P \in V$. By Lemma 5.1 of [4], $D x \in Q(P / A)$ if $x \in Q(P / A)$; hence $D x \in Q(V / A)$ if $x \in Q(V / A)$. If $t$ is a regular parameter of $Q(V / A)$, the derivation $t D$ has a zero at $V$. Since $\omega$ does not have a pole at $V$, we conclude that $t a=\omega(t D) \in \mathfrak{B}(V / A)$, so that $a \in Q(V / A)$. This having to be true for any $V$, it follows that $a \in k$. If then $\left\{D_{i}\right\},\left\{\omega_{i}\right\}$ have the previous meaning, and $\omega D_{i}=a_{i} \in k$, we necessarily have $\omega=\sum_{i} a_{i} \omega_{i} \in D_{1}(A)$, as claimed. The existence of an absolute basis for $D_{1}(A)$ is an immediate consequence of this fact, and of 1.8, Q.E.D.

Before proceeding any further, we remark that the differentials on a curve, in the language of [6], can be identified with our differentials thanks to Theorem 6 of Chapter VI of [6]; also the definitions of "first kind" and "second kind" are equivalent (see $\$ 8$, Chapter VI of [6]). And it is apparent that if $C$ is an absolutely irreducible curve without singularities over $k, D_{1}(C)$ has an absolute basis, and a representative of an absolute basis of $D_{2}(C) / D_{e}(C)$ exists. $D_{1}(C)$ is a $k$-module of order $g=$ genus of $C$, while $D_{2}(C) / D_{e}(C)$ is a $k$-module of order $2 g$ if $k$ has characteristic zero. In the latter case, the $k$-modules $D_{2}(C) / D_{1}(C)+D_{e}(C)$ and $D_{1}(C)$ are dual to each other, by Theorem 8 of Chapter VII of [6]; the duality operation is induced by the operation $j\left(\omega_{2}, \omega_{1}\right)$, for $\omega_{i} \in D_{i}(C)$.

2.2. Lemma. Let $A$ be an $n$-dimensional nonsingular abelian variety over the algebraically closed field $k$; then each semi-invariant differential on $A$ is of the second kind. If $k$ has characteristic zero, the following statement is also true: let $\omega$ be a closed differential on $A$, such that for any $(n-1)$-dimensional irreducible subvariety $V$ of $A$ there exists an $f \in k(A)$ for which $\omega-d_{A} f$ is regular at $V$; then $\omega$ is semi-invariant, hence of the second kind. In particular, every element of $D_{2}(A)$ is semi-invariant.

Proof. Let $\omega$ be a semi-invariant differential on $A$, and let $U$ be an irreducible subvariety of $A$. Since $\omega$ has finitely many poles by 1.4 , for a suitable $P \in A$ the differential $\sigma_{P} \omega$ does not have a pole at $U$; as $\sigma_{P} \omega \sim \omega$, we conclude that $\omega$ is of the second kind.

In order to prove the second statement, assume $k$ to have characteristic zero, and denote by $D^{\prime}$ the set of the closed differentials on $A$ having the property described in the statement of the lemma. For any differential $\omega$ on $A$, let $p(\omega)$ be its polar variety, that is, the join of all its poles, and let $e(\omega)$ denote the intersection of all the $p\left(\omega+d_{A} f\right)$ when $f$ ranges in $k(A)$; if $\omega$ is closed, we have $\omega \in D^{\prime}$ if and only if $\operatorname{dim} e(\omega)<n-1$. If $P \in A$, we have $e\left(\sigma_{P} \omega\right)=\sigma_{P} e(\omega)$, so that $\sigma_{P} \omega \in \mathscr{D}^{\prime}$ if and only $\omega \in \mathfrak{D}^{\prime}$. 
Since $A$ is the homomorphic image of a jacobian variety, there exist a curve $C$ on $k$, without singularities, and a rational mapping $\phi$ of $C$ into $A$, such that, for each $P \in A$, no proper group-subvariety of $A$ contains $\sigma_{P} \phi C$, $\phi C$ denoting the curve on $A$ on which $\phi$ operates.

We shall now consider copies $A, A_{1}, A_{2}$ of $A$, and assume $k\left(A_{2}\right) \subseteq k\left(A \times A_{1}\right)$ as prescribed by the rational mapping of $A \times A_{1}$ onto $A_{2}$ which gives the law of composition on $A$; as usual, and unless stated otherwise, if $\omega$ is any entity related to $A, \omega_{i}$ shall denote the copy of $\omega$ similarly related to $A_{i}$. Let $\{x\},\{y\}$ be n.h.g.p. of $A, A_{1}$ respectively, copies of each other; set $H=k\left(A_{1}\right), L=k(A)$, $K=k(C)$; let $X$ be the point of $A_{H}$ at which the co-ordinate $x_{i}$ assumes the value $y_{i}$, and let $Z$ be the point of $\left(A_{i}\right)_{L}$ at which the co-ordinate $y_{i}$ assumes the value $x_{i}$; set also $Y=\phi\{C\} \in A_{K}$.

Quite in general, if $\omega$ is a closed differential on the product $U \times V$ of two irreducible varieties, $\omega$ can be written uniquely as the sum $\omega_{U}+\omega_{V}$ of two differentials on $U \times V$, such that $\omega_{U} D=0$ for any derivation $D$ of $k(U \times V)$ over $k(U)$, while $\omega_{V}$ is similarly related to $V$; we shall say that $\omega_{U}$ is the $U$-component of $\omega$, and shall use this notation throughout this proof; $\omega_{U}$ is not, generally, a closed differential. Under the same conditions, let $\omega_{U}^{\prime}$ be the differential on $U_{k(V)}$ such that $\omega_{U}^{\prime} D=\omega_{U} D=\omega D$ for any derivation $D$ on $U_{k(V)}$; then $\omega_{U}^{\prime}$ is closed, and will be called the $U_{k(V)}$-component of $\omega$.

We shall now consider a differential $\omega \in D^{\prime}$, and shall denote by $\Omega$ the extension of $\omega_{2}$ on $A \times A_{1}$; we shall also write $\Omega=\Omega_{A}+\Omega_{A_{1}}$, these being the $A$-component and the $A_{1}$-component of $\Omega$; let $\omega^{*}$ be the $A_{H}$-component of $\Omega$. Then we have $\omega^{*}=\sigma_{X}^{-1} \omega_{H}$, so that $e\left(\omega^{*}\right)=\sigma_{X}^{-1} e\left(\omega_{H}\right)$; and of course, any component of $e\left(\omega_{H}\right)$ is a subvariety of the extension over $H$ of a component of $e(\omega)$. Since $e\left(C_{H} \times \omega^{*}\right)=C_{H} \times e\left(\omega^{*}\right)$, the previous result implies that the intersection of $\phi_{H}$ and $e\left(C_{H} \times \omega^{*}\right)$, on $C_{H} \times A_{H}$, is empty. Since each point of $\phi_{H}$ is simple on $C_{H} \times A_{H}$ and on $\phi_{H}, 1.4$ and 1.5 apply to this case, and yield that $\phi_{H} \cap\left(C_{H} \times \omega^{*}\right)$ is a differential of the second kind on $\phi_{H}$; as $k(\phi)$ can be identified with $K$, there exists a unique differential $\alpha^{*}$ on $C_{H}$, of the second kind, such that $\left(\alpha^{*} \times A_{H}\right) \cap \phi_{H}=\phi_{H} \cap\left(C_{H} \times \omega^{*}\right)$. Let $\left\{\alpha_{1}, \cdots, \alpha_{r}\right\}$ be a representative of an absolute basis of $D_{2}(C) / D_{e}(C)$; then, for suitable elements $z_{i} \in H$, we have $\alpha^{*} \sim \sum_{i} z_{i}\left(\alpha_{i}\right)_{H}$; this means that there exists an $f \in k\left(C \times A_{1}\right)$

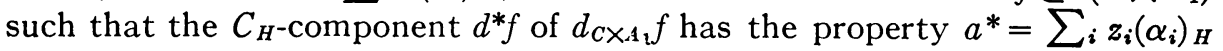
$+d^{*} f$. Denote by $\alpha_{C}$ the differential on $C \times A_{1}$ such that $\alpha_{C} D=\alpha^{*} D$ for any derivation $D$ on $C_{H}$, while $\alpha_{C} D=0$ for any derivation $D$ on $\left(A_{1}\right)_{K}$; then

$$
\left(\alpha_{C} \times A\right) \cap\left(\phi \times A_{1}\right)=\left(\phi \times A_{1}\right) \cap\left(C \times \Omega_{A}\right),
$$

and $\alpha_{C}=\sum_{i} z_{i}\left(\alpha_{i} \times A_{1}\right)+\left(d_{C \times A_{1}} f\right)_{C}$.

We want to prove that $\left(\phi \times A_{1}\right) \cap\left(C \times \Omega_{A}\right)$ has no pole of the type $\phi \times U_{1}$, with $U$ an $(n-1)$-dimensional irreducible subvariety of $A$. In fact, consider a pole of this type; then, for any $P \in U, \phi \times P_{1}$ is a subvariety of a pole of $\left(\phi \times A_{1}\right) \cap\left(C \times \Omega_{A}\right)$, hence, by 1.5, also of a pole of $C \times \Omega_{A}$; if $V$ is any pole of 
$\omega, V^{\prime}=\sigma_{X}^{-1} V_{H}$ is a pole of $\omega^{*}$, and $V^{\prime \prime}=D_{V^{\prime}, A_{1}}$ is a pole of $\Omega_{A}$, so that $C \times V^{\prime \prime}$ is a pole of $C \times \Omega_{A}$. Conversely, each pole of $C \times \Omega_{A}$ is of this type, because it operates on the whole $A_{1}$; consequently, if $\phi \times P_{1}$ is a subvariety of a pole of $C \times \Omega_{A}, Y \times\left(P_{1}\right)_{K}$ will be a subvariety of a $V_{K}^{\prime \prime}$, where $V^{\prime \prime}$ is of the previous type; this means that $Y$ and $\left(P_{1}\right)_{K}$ correspond to each other in the algebraic correspondence $V_{K}^{\prime \prime}$; but $V^{\prime \prime}\left[P_{1}\right]=\sigma_{P}^{-1} V$ (see $\S 1$ of $[4]$; in the notation of that section, after exchanging $G_{1}, G_{2}, G_{3}$ with, respectively, $A_{1}, A_{2}, A, V^{\prime}$ would be denoted by $\Gamma_{V}$, and $V^{\prime \prime}$ by $\left.T_{V}\right)$, so that $Y \in\left(\sigma_{P}^{-1} V\right)_{K}$, or $\sigma_{P_{K}} Y \in V_{K}$; this, in turn, implies $\sigma_{P} \phi C \subseteq V_{K}$ for any $P \in U$, or $\sigma_{Q} U=V$ for each $Q \in \phi C$. But this is impossible, since it implies that, for a fixed $R \in \phi C, \sigma_{R}^{-1} \phi C$ belongs to the proper group-subvariety of $A$ consisting of the $P \in A$ such that $\sigma_{P} U=U$. It is thus proved that no pole of $\left(\phi \times A_{1}\right) \cap\left(C \times \Omega_{A}\right)$ is of the type $\phi \times U_{1}$; hence, by $2.3,\left(\alpha_{C} \times A\right) \cap\left(\phi \times A_{1}\right)$ has the same property.

Now, for any $(n-1)$-dimensional irreducible subvariety $U_{1}$ of $A_{1}$, let $\left\{\xi_{1}, \cdots, \xi_{n}\right\}$ be a set of uniformizing parameters at $U_{1}$ on $A_{1}$; for a given element $z$ of $K$ but not of $k$, there exists an element $g \in k\left(C \times A_{1}\right)$ such that $\alpha_{C}=g\left(d_{C} z \times A_{1}\right)=g d_{C \times A_{1}} z ;$ hence, $\quad\left(\alpha_{C} \times A\right) \cap\left(\phi \times A_{1}\right)=g\left(d_{\phi} z \times A_{1}\right)=g d_{\phi \times A_{1}} z$, where $z$ is now considered as an element of $k(\phi)$ in the identification $k(\phi)=K$. A set of uniformizing parameters at $\phi \times U_{1}$ on $\phi \times A_{1}$ is $\left\{z, \xi_{1}, \cdots, \xi_{n}\right\}$, so that, by 1.2 , the fact that $\phi \times U_{1}$ is not a pole of $\left(\alpha_{C} \times A\right)$ $\cap\left(\phi \times A_{1}\right)$ means that $C \times U_{1}$ is not a pole of $g$; as a consequence, and for the same reason, $C \times U_{1}$ is not a pole of $\alpha_{C}$, and this is valid for each $U_{1}$. We contend that, as a consequence, $z_{i} \in k$ for each $i$. In fact, if it is not so, after setting $d^{*} f=f^{\prime} d^{*} z$ for $f^{\prime} \in k\left(C \times A_{1}\right)$, let $U_{1}$ be a pole of some $z_{i}$, and let $p \in \mathfrak{P}\left(U_{1} / A_{1}\right)$ be such that $p z_{i}, p f^{\prime} \in Q\left(C \times U_{1} / C \times A_{1}\right)$, one of them at least not being in $\mathfrak{B}\left(C \times U_{1} / C \times A_{1}\right)$. Then

$$
0=p \alpha_{C} \cap\left(C \times U_{1}\right)=\sum_{i}\left(\pi\left(p z_{i}\right)\right)\left(\alpha_{i} \times U_{1}\right)+\left(\pi\left(p f^{\prime}\right)\right)\left(d_{C \times U_{1}} z\right)_{C},
$$

where $\pi$ denotes reduction of $Q\left(C \times U_{1} / C \times A_{1}\right) \bmod \mathfrak{B}\left(C \times U_{1} / C \times A_{1}\right)$; hence,

$$
\sum\left(\pi\left(p z_{i}\right)\right)\left(\alpha_{i}\right)_{M}+\left(\pi\left(p f^{\prime}\right)\right) d_{C_{M}} z=0,
$$

where $M=k\left(U_{1}\right)$. Since the $\left(\alpha_{i}\right)_{M}$ represent an $M$-independent basis for $D_{2}\left(C_{M}\right) / D_{e}\left(C_{M}\right)$, we conclude that $\pi\left(p z_{i}\right)=\pi\left(p f^{\prime}\right)=0$, against the hypothesis. We have thus shown that $z_{i} \in k$ for each $i$, so that there exists an element $\alpha=\sum_{i} z_{i} \alpha_{i}$ of $\mathfrak{D}_{2}(C)$, and 2.3 can be written as

$$
\left[\alpha \times A \times A_{1}+d_{C \times A \times A_{1}} f-C \times \Omega\right]_{A \times C} \cap\left(\phi \times A_{1}\right)=0 .
$$

This also means that the $\phi$-component of

$$
\left[\alpha \times A \times A_{1}+d_{C \times A \times A_{1}} f-C \times \Omega\right] \cap\left(\phi \times A_{1}\right)
$$

is zero. As this is a closed differential, it must necessarily be of the type $\phi \times \beta$, with $\beta$ a closed differential on $A_{1}$. The $\left(A_{1}\right)_{K}$-component of the above differen- 
tial is then $\beta_{K}=\left(d^{\prime} f-\Omega_{K}\right) \cap\left(Y \times\left(A_{1}\right)_{K}\right)$, where $d^{\prime}=d_{\left(A_{1) K}\right.}$, or also $\beta_{K}$ $=\left(d^{\prime} f-\Omega_{A_{1}}\right) \cap\left(Y \times\left(A_{1}\right)_{K}\right)$. For any invariant derivation $D$ on $A$, denote by $D_{1}$ also the extension of $D_{1}$ over $K$, or over $k(C \times A)$; then $D_{1}$ is regular at $Y \times\left(A_{1}\right)_{K}$, and we have $\left[\left(d^{\prime} f-\Omega_{A_{1}}\right) \cap\left(Y \times\left(A_{1}\right)_{K}\right)\right] D_{1}=D_{1} f-\pi\left[\left(\sigma_{z}^{-1}\left(\omega_{1}\right)_{L}\right) D_{1}\right]$, where $\pi$ now denotes the homomorphic mapping of $Q\left(Y \times\left(A_{1}\right)_{K} / A_{K} \times\left(A_{1}\right)_{K}\right)$ onto $K\left(\left(A_{1}\right)_{K}\right)$ whose kernel is $\mathfrak{P}\left(Y \times\left(A_{1}\right)_{K} / A_{K} \times\left(A_{1}\right)_{K}\right)$; the last expression coincides with

$$
D_{1} f-\pi\left[\sigma_{Z}^{-1}\left(\left(\omega_{1}\right)_{L} D_{1}\right)\right]=D_{1} f-\sigma_{Y_{1}}^{-1}\left(\omega_{1} D_{1}\right)=D_{1} f-\left(\sigma_{Y_{1}}^{-1}\left(\omega_{1}\right)_{K}\right) D_{1} ;
$$

thus, $\beta_{K}=d^{\prime} f-\sigma_{Y_{1}}^{-1}\left(\omega_{1}\right)_{K}$. Now, for any $P \in C$, the above reasoning can be repeated, and it gives $\beta \sim\left(\sigma_{Q}^{-1} \omega\right)_{1}$ if $Q=\phi[P]$. If $S$ is a fixed point of $\phi C$, and $C^{\prime}=\sigma_{\mathcal{S}}^{-1} \phi C$, let $Q_{1}, \cdots, Q_{n} \in C^{\prime}$; then $\omega \sim \sigma_{Q_{1}}^{-1} \omega$, hence $\omega \sim \sigma_{Q_{2}}^{-1} \omega \sim \sigma_{Q_{1} Q_{2}}^{-1} \omega$, etc., so that $\omega \sim \sigma_{R}^{-1} \omega$ for $R=Q_{1} Q_{2} \cdots Q_{n}$; but as the $Q_{i}$ range over $C^{\prime}, R$ ranges over $A$, so that $\omega \sim \sigma_{R} \omega$ for any $R \in A$, and $\omega$ is semi-invariant, Q.E.D.

2.4. Lemma. Let $A$ be an n-dimensional nonsingular jacobian variety over the algebraically closed field $k$ of characteristic zero; then $D_{2}(A) / D_{e}(A)$ is a $k$ module of order $2 n$, and there exists a representative of an absolute basis of $i$.

$\operatorname{Proof}\left({ }^{2}\right)$. Let $A$ be the jacobian of a curve $C$ over $k$, without singular points. Let $C_{1}, \cdots, C_{n}$ be copies of $C$, and set $V=C_{1} \times \cdots \times C_{n}$. Then, by [9], $k(A)$ is the set of the elements of $k(V)$ which are invariant for all the elements of the group $G$ of the automorphisms of $k(V)$ over $k$ which permute the $k\left(C_{i}\right)$ in all possible manners. Since there exists a representative of an absolute basis of $D_{2}(C) / D_{e}(C), 1.9$ implies that there exists a representative of an absolute basis of $\mathscr{D}_{2}(V) / \mathscr{D}_{e}(V)$, and also that $\mathscr{D}_{2}(V) / \mathscr{D}_{e}(V)$ is a $k$ module of order $2 n^{2}$. Let $\omega \in D_{2}(A)$, and let $\omega^{\prime}$ be its extension on $V$; then $\omega^{\prime}$ is a closed differential, invariant for each element of $G$; we contend that $\omega^{\prime}$ is of the second kind. Let $W$ be a nonempty irreducible subvariety of $V$; by Theorem 6 of [9], if $\phi$ is the rational mapping of $V$ onto $A$ generated by the embedding $k(A) \subseteq k(V)$, the variety $U$ on which $\phi[W]$ operates is such that $Q(U / A) \subseteq Q(W / V)$. There exists a $\omega_{1} \sim \omega$ regular at $U$, and the extension $\omega_{1}^{\prime}$ of $\omega_{1}$ on $V$ is regular at $W$ by 1.2 , and clearly $\omega_{1}^{\prime} \sim \omega^{\prime}$. Hence $\omega^{\prime}$ is of the second kind. Conversely, let $\omega^{\prime}$ be a closed differential of the second kind on $V$, invariant for each element of $G$. If $x_{1}, \cdots, x_{n} \in k(A)$, and $k(A)$ is a finite extension of $k(x)$, also $k(V)$ is a finite extension of $k(x)$; write $\omega^{\prime}=\sum_{i} a_{i} d_{V} x_{i}$, $a_{i} \in k(V)$. Then each $a_{i}$ is invariant for each element of $G$, and therefore $a_{i} \in k(A)$, and $\omega^{\prime}$ is the extension on $V$ of $\omega=\sum_{i} a_{i} d_{A} x_{i}$. We contend that $\omega$ has the following property: for any $(n-1)$-dimensional irreducible subvariety $W$ of $A$, there exists an $f \in k(A)$ such that $\omega-d_{A} f$ is regular at $W$. In order to prove the assertion, we consider the distinct components $W_{1}, W_{2}, \cdots$ of

(2) (Added November 4, 1955). The author is indebted to the referee of Amer. J. Math. for pointing out an error in the proof which the author had originally submitted for this lemma. 
the subvariety of $V$ on which $\phi[W]$ operates; they are all $(n-1)$-dimensional, and conjugate to each other under the transformations of $G$ (see $\S 3$ of [4]). Since $\omega^{\prime}$ is of the second kind, there exists an $f_{1} \in k(V)$ such that $\omega^{\prime}-d_{V} f_{1}$ is regular at $W_{1}$; moreover, the $Q\left(W_{j} / V\right)$ are disjoint valuation rings in the sense of Krull, and therefore, by the independence theorem, it is possible to select an $f^{\prime} \in \bigcap_{j \neq 1} Q\left(W_{j} / V\right)$ such that $f_{1}-f^{\prime} \in Q\left(W_{1} / V\right)$. Let $m$ be the number of times $W_{1}$ (hence any $W_{j}$ ) appears among the conjugates of $W_{1}$, under the transformations $g_{1}, g_{2}, \cdots$ of $G$, and set $f=m^{-1} \sum_{j} g_{j} f^{\prime}$; then, since $g_{j} \omega^{\prime}=\omega^{\prime}$ for each $j, \omega^{\prime}-d_{V} f$ is regular at each $W_{j}$; but $f \in k(A)$, so that $\omega^{\prime}-d_{V} f$ is the extension on $V$ of $\omega-d_{A} f$; since $Q(W / A)=k(A) \cap Q\left(W_{1} / V\right)$, we conclude that $\omega-d_{A} f$ is regular at $W$, as claimed. Thus, by $2.2, \omega \in D_{2}(A)$.

We have thus established a 1-1 correspondence $\omega \rightarrow \omega^{\prime}$ between $D_{2}(A)$, and the $k$-module $S$ of the elements of $D_{2}(V)$ which are invariant under the transformations of $G$. The $k$-module $S$ consists, by 1.9 , of all the differentials of the type

$$
\sum_{i=1}^{n} C_{1} \times \cdots \times C_{i-1} \times \omega_{i}^{*} \times C_{i+1} \times \cdots \times C_{n}+d_{V} f,
$$

where $f \in k(A)$ and $\omega_{i}^{*}$ is the copy on $C_{i}$ of a $\omega^{*} \in D_{2}(C)$; clearly, $\mathrm{s} / \mathrm{s} \cap D_{e}(V)$ has order $2 n$, so that $D_{2}(A) / D_{e}(A)$ has order $2 n$. As a consequence, there exists an absolute basis of it, Q.E.D.

REMARK. In the notation of the preceding proof, and for a $\omega \in \mathfrak{D}_{2}(A)$, let $\omega^{\prime}$ be the extension of $\omega$ on $V$, and let $\omega^{*} \in D_{2}(C)$ be such that

$$
\omega^{\prime} \sim \sum_{i} C_{1} \times \cdots \times C_{i-1} \times \omega_{i}^{*} \times C_{i+1} \times \cdots \times C_{n} ;
$$

then the mapping $\omega \rightarrow \omega^{*}$ induces an isomorphism between $\mathscr{D}_{2}(A) / D_{e}(A)$ and $D_{2}(C) / D_{e}(C)$, in which $D_{1}(A)$ and $D_{1}(C)$ map into each other.

Let $A$ be an $n$-dimensional nonsingular abelian variety over the algebraically closed field $k$ of characteristic zero; let $C$ be a curve without singularities over $k$, and let $\lambda$ be a rational mapping of $C$ into $A$. For any $\omega \in D_{2}(A)$, we shall consider the element $\lambda^{*}(\omega) \in D_{2}(C)$ such that $\left(\lambda^{*}(\omega) \times A\right) \cap \lambda$ $=(C \times \omega) \cap \lambda$, if these expressions have a meaning. Then, $\lambda^{*}$ induces a homomorphic mapping of $D_{2}(A) / D_{e}(A)$ into $D_{2}(C) / D_{e}(C)$, such that $D_{1}(A)$ maps into $D_{1}(C)$. If, in particular, $A$ is the jacobian of $C$, and $\lambda$ is a canonical mapping of $C$ into $A, \lambda^{*}(\omega)$ is the differential on $C$ denoted by $\omega^{*}$ in the preceding remark (but for an exact differential); accordingly, in this particular case, the mapping $\lambda^{*}: \omega \rightarrow \lambda^{*}(\omega)$ induces an isomorphism between $D_{2}(A) / D_{e}(A)$ and $D_{2}(C) / D_{e}(C)$, in which $D_{1}(A)$ and $D_{1}(C)$ map into each other.

In general, $\lambda$ can be extended to a homomorphism $\alpha$ of the jacobian $J$ of $C$ into $A$; in other words, $\lambda=\alpha \phi$, if $\phi$ is a canonical mapping of $C$ into $J$. If, in particular, $\alpha$ maps $J$ onto $A$, then $\lambda^{*}(\omega)=\phi^{*}\left(\omega^{\prime}\right)$, if $\omega^{\prime}$ is the extension of $\omega$ on $J$, when $k(A) \subseteq k(J)$ as prescribed by $\alpha$. Clearly, $\omega^{\prime} \in D_{2}(J)$ if $\omega \in D_{2}(A)$; and of course $\omega^{\prime} \in D_{1}(J)$ if $\omega \in \mathscr{D}_{1}(A)$; also, if $\omega \in \mathscr{D}_{2}(A)$ and $\omega^{\prime} \in \mathscr{D}_{1}(J)$, then 
necessarily $\omega \in D_{1}(A)$, by 2.1 . Consequently, if $\alpha$ maps $J$ onto $A$, or, equivalently, if, for any $P \in A$, no proper abelian subvariety of $A$ contains $\sigma_{P} \lambda C$, $\lambda *$ induces an isomorphic mapping of $D_{2}(A) / D_{e}(A)$ into $D_{2}(C) / D_{e}(C)$, in which $D_{1}(A)$ maps into $D_{1}(C)$, and in which no element of $D_{2}(A) / D_{e}(A)$, but not of $D_{1}(A)$, maps into an element of $D_{1}(C)$.

Now, for an arbitrary $A$, let $\lambda, \mu$ be rational mappings of $C$ into $A$. We shall denote by $\lambda+\mu$ the rational mapping $\nu$ of $C$ into $A$ such that $\nu[C]$ $=(\lambda[C])(\mu[C])$. We contend that $(\lambda+\mu)^{*} \sim \lambda^{*}+\mu^{*}$, in the sense that $(\lambda+\mu)^{*}(\omega) \sim \lambda^{*}(\omega)+\mu^{*}(\omega)$ for any $\omega \in D_{2}(A)$. In fact, let $A_{1}, A_{2}, A_{3}$ be copies of $A$, and let $D$ be the rational mapping of $A_{1} \times A_{2}$ onto $A_{3}$ which gives the law of composition on $A$. Let $\omega_{1}, \omega_{2}, \omega_{3}$ be the copies of $\omega$, on, respectively, $A_{1}, A_{2}, A_{3}$. We shall denote by $\lambda_{1}, \mu_{2}, \nu_{3}$ the copies of, respectively, $\lambda, \mu, \nu$ $=\lambda+\mu$ which map $C$ on, respectively, $A_{1}, A_{2}, A_{3}$. Then $\left(\omega_{1} \times A_{2} \times A_{3}\right.$ $\left.+\omega_{2} \times A_{1} \times A_{3}-\omega_{3} \times A_{1} \times A_{2}\right) \cap D \sim 0$, since $\omega$ is semi-invariant, by 2.2 . Also, $\theta=\left(\lambda_{1} \times A_{2} \times A_{3}\right) \cap\left(\mu_{2} \times A_{1} \times A_{3}\right) \cap\left(\nu_{3} \times A_{1} \times A_{2}\right)$ is a curve on $D \times C$ which, if considered as an algebraic correspondence between $C$ and $D$, operates on the whole $C$. As a consequence, we have:

$$
\begin{aligned}
& {\left[\left(\lambda^{*}(\omega)+\mu^{*}(\omega)-\nu^{*}(\omega)\right) \times A_{1} \times A_{2} \times A_{3}\right] \cap \theta } \\
&=\left\{\left[\left(\lambda^{*}(\omega) \times A_{1}\right) \cap \lambda_{1}\right] \times A_{2} \times A_{3}\right\} \cap \theta \\
&+\left\{\left[\left(\mu^{*}(\omega) \times A_{2}\right) \cap \mu_{2}\right] \times A_{1} \times A_{3}\right\} \cap \theta \\
&-\left\{\left[\left(\nu^{*}(\omega) \times A_{3}\right) \cap \nu_{3}\right] \times A_{1} \times A_{2}\right\} \cap \theta \\
&=\left\{\left[\left(C \times \omega_{1}\right) \cap \lambda_{1}\right] \times A_{2} \times A_{3}\right\} \cap \theta+\left\{\left[\left(C \times \omega_{2}\right) \cap \mu_{2}\right] \times A_{1} \times A_{3}\right\} \cap \theta \\
&-\left\{\left[\left(C \times \omega_{3}\right) \cap \nu_{3}\right] \times A_{1} \times A_{2}\right\} \cap \theta \\
&= {\left[C \times\left(\omega_{1} \times A_{2} \times A_{3}+\omega_{2} \times A_{1} \times A_{3}-\omega_{3} \times A_{1} \times A_{2}\right)\right] \cap \theta } \\
&= {[C \times(\cdots)] \cap(C \times D) \cap \theta=\{C \times[(\cdots) \cap D]\} \cap \theta \sim 0 . }
\end{aligned}
$$

Consequently, since $\theta$ operates on the whole $C$, we conclude that $\lambda^{*}(\omega)$ $+\mu^{*}(\omega)-\nu^{*}(\omega) \sim 0$, as claimed.

2.5. Lemma. Let $A$ be an abelian subvariety of the nonsingular abelian variety $B$ over the algebraically closed field $k$ of characteristic zero; then $D_{1}(A)$ is the set of the $\omega \cap A$, when $\omega$ ranges in $D_{1}(B)$; also, $D_{2}(A)$ is the set of the $\omega \cap A$ when $\omega$ ranges over all the elements of $D_{2}(B)$ which do not have a pole at $A$.

Proof. $B$ is isogenous to $A \times C$, for a suitable nonsingular abelian variety $C$; assume $k(A \times C) \subseteq k(B)$ as prescribed by a homomorphism $\alpha$ of $B$ onto $A \times C$ of positive degree, such that $\alpha A=A$. For any $\omega \in D_{2}(A \times C)$, let $\omega^{\prime}$ be its extension on $B$; then the mapping $\omega \rightarrow \omega^{\prime}$ establishes an isomorphism between $D_{2}(A \times C) / D_{e}(A \times C)$ and $\mathscr{D}_{2}(B) / D_{e}(B)$, in which $D_{1}(A \times C)$ and $\mathscr{D}_{1}(B)$ correspond to each other. Thus it is sufficient to prove the lemma when $B$ is replaced by $A \times C$, and $A$ by $A \times E_{C}$. But in this case the lemma is a consequence of 1.9 , Q.E.D. 
2.6. Lemma. Let $\alpha$ be a homomorphism of an abelian variety $A$ over the algebraically closed field $k$, into an abelian variety $B$ over $k$, and, for a prime $l$ not equal to the characteristic of $k$, let $M$ be an l-adic matrix related to $\alpha$ as in Theorem 14 of [9]. Then the rank of $M$ is twice the dimension of $\alpha A$.

Proof. If $\operatorname{dim} \alpha A=n$, there is an $n$-dimensional abelian subvariety $C$ of $A$ such that $\alpha C=\alpha A$; we may replace $A$ with an isogenous variety $C \times C^{\prime}$ without affecting ranks and dimensions, by Proposition 13 of [9]. Accordingly, we shall assume $A=C \times C^{\prime}$, and $\alpha\left(C \times E_{C^{\prime}}\right)=\alpha A$, while $\alpha\left(E_{C} \times C^{\prime}\right)$ $=E_{\alpha A}$. After a suitable selection of $l$-adic co-ordinates on $C \times C^{\prime}, M$ assumes the form $\left(M_{1} \mid M_{2}\right)$, where $M_{1}, M_{2}$ are the $l$-adic matrices related to, respectively, $\left[\alpha ; C \times E_{C^{\prime}}, B\right]$ and $\left[\alpha ; E_{C} \times C^{\prime}, B\right]$. But then, by Proposition 13 and by Corollary 3 to Theorem 33 of [9], $M_{1}$ has rank $2 n$, and $M_{2}=0$, Q.E.D.

Now, let $J$ be the jacobian of a curve $C$, without singularities, over the algebraically closed field $k$; by Corollary 2 to Theorem 22 of [9], there is an isomorphism $D \rightarrow \lambda$ between the ring of the classes of algebraic correspondences $D$ between $C$ and a copy $C^{\prime}$ of $C$, and the ring $R$ of the endomorphisms $\lambda$ of $J$. The interchange of $C$ with $C^{\prime}$ induces an involution $\lambda \rightarrow \lambda^{\prime}$ in $R$, which in turn induces an involution in the algebra $a$ of the endomorphisms of $J$ (=smallest algebra containing $R$ ). This involution will be called the Rosati involution (see [7]).

2.7. Lemma. With these notations, $\lambda$ and $\lambda^{\prime}$ have the same characteristic polynomial; if $l$ is a prime different from the characteristic of $k$, and if $L, L^{\prime}$ are the $l$-adic matrices related to $\lambda, \lambda^{\prime}$ respectively, then $L$ and $L^{\prime}$ have the same rank and the same characteristic polynomial.

Proof $\left({ }^{3}\right)$. We shall denote by $Q^{*}$ the algebra of the $l$-adic matrices related to the elements of $a$. Let $B$ be an abelian variety isogenous to $J$; by $\$ 54$ of [9], if $\mu$ is a homomorphism of $B$ onto $J$, the correspondence $\lambda \rightarrow \lambda_{1}=\mu^{-1} \lambda \mu$ is an isomorphism between $Q$ and the algebra $B$ of the endomorphisms of $B$; if $L, L_{1}, M$ are the $l$-adic matrices related to, respectively, $\lambda, \lambda_{1}, \mu$, we have $L_{1}=M^{-1} L M$, and this proves that the isomorphism $L \rightarrow L_{1}$ preserves the characteristic polynomial and the rank. Moreover, if $\lambda_{1}^{\prime}=\mu^{-1} \lambda^{\prime} \mu$, the mapping $\lambda_{1} \rightarrow \lambda_{1}^{\prime}$ is an involution in $B$. Theorem 25 of [9] implies that $\lambda^{\prime} \lambda=0$ if and only if $\lambda=0$; consequently, $\lambda_{1}^{\prime} \lambda_{1}=0$ if and only if $\lambda_{1}=0$. Now, by Theorem 28 of [9], we can select $B$ to be of the type $B_{1} \times \cdots \times B_{r}$, where each $B_{i}$ is the direct product of simple abelian varieties, isomorphic to each other, while no abelian subvariety of $B_{i}$ (for $i=1, \cdots, r$ ) is isogenous to any abelian subvariety of $B_{j}$ if $j \neq i$. If $B_{i}$ is the algebra of the endomorphisms of $B_{i}$, by Theorem 29 of [9] $B_{i}$ is a simple algebra, and $B$ is the direct sum of $B_{1}, \cdots, B_{r}$; more precisely, this is so after identification of an endomorphism

(3) This result is also an immediate consequence of the last formula of $\$ 76$ of [9]; see however the remark which follows this proof. 
$\nu$ of $B_{i}$ with the endomorphism $\nu^{\prime}$ of $B$ such that $\nu^{\prime}\left[P_{1} \times \cdots \times P_{\mathbf{r}}\right]$ $=E_{B_{1}} \times \cdots \times E_{B_{i-1}} \times \nu P_{i} \times E_{B_{i+1}} \times \cdots \times E_{B_{r}}$, for $P_{j} \in B_{j}$.

Let $B_{i}^{\prime}$ be the transform of $B_{i}$ in the involution ' on $B$; then $B_{i}^{\prime}$ must coincide with some $B_{j}$, since the decomposition of a semi-simple algebra as direct sum of simple algebras is unique. Should $j$ be different from $i$, an element $\lambda_{1} \in B_{i}$ would have the property that $\lambda_{1}^{\prime} \lambda_{1}\left[P_{1} \times \cdots \times P_{r}\right]$ $=\lambda_{1}^{\prime}\left[E_{B_{1}} \times \cdots \times E_{B_{i-1}} \times \lambda_{1} P_{i} \times E_{B_{i+1}} \times \cdots \times E_{B_{r}}\right]=E_{B}$, or $\lambda_{1}^{\prime} \lambda_{1}=0$, or finally $\lambda_{1}=0$. Consequently, $B_{i}^{\prime}=B_{i}$, and the involution ' induces an involution in each $B_{i}$. Now, for a suitable choice of $l$-adic co-ordinates in $B$, the $l$-adic matrix $L_{1}$ related to $\lambda_{1}=\sum_{i} \lambda_{1 i}\left(\lambda_{1 i} \in B_{i}\right)$ can be written in the form

$$
\left(\begin{array}{llll}
L_{11} & & & \\
& L_{12} & & \\
& & \ddots & \\
& & L_{1 r}
\end{array}\right) \text {, }
$$

where $L_{1 i}$ is the $l$-adic matrix related to $\lambda_{1 i}$. When $\lambda_{1}$ ranges in $B, L_{1 i}$ ranges in the algebra $B_{i}^{*}$ of the $l$-adic matrices related to the elements of $B_{i}$; the involution ' induces an involution in each $B_{i}{ }^{*}$, and $B_{i}{ }^{*}$ is a simple subring of the algebra $\mathfrak{T H}_{i}$ of all the matrices, with $l$-adic elements, of the same order as $L_{1 i}$. Therefore, by a result on algebras $\left({ }^{4}\right)$, there exists an element $N \in \Re_{i}$ such that, for each $L_{1 i} \in B_{i}^{*}, L_{1 i}^{\prime}=N^{-1}\left(L_{1 i}\right)_{-1} N$, where ${ }_{-1}$ denotes transposition of matrices. Thus $L_{1 i}^{\prime}$ and $L_{1 i}$ have the same characteristic polynomial, also the same rank; hence $L_{1}$ and $L_{1}^{\prime}$, or also $L$ and $L^{\prime}$, have the same characteristic polynomial and the same rank, Q.E.D.

REMARK. It may be noted that the preceding proof, and the preceding lemma, are valid not only for the Rosati involution on a jacobian variety, but also for any reciprocity ' onto itself of the algebra $Q$ of the endomorphisms of an abelian variety, provided such reciprocity has the property that $\lambda^{\prime} \lambda=0$ implies $\lambda=0$ for $\lambda \in Q$.

2.8. THEOREM. Let $A$ be a nonsingular $n$-dimensional abelian variety over the algebraically closed field $k$. Then the semi-invariant differentials on $A$ are all of the second kind. If, in addition, $k$ has characteristic zero, $D_{2}(A) / D_{e}(A)$ is a $k$-module of order $2 n$, and there exists a representative of an absolute basis of $i t$; also, each element of $D_{2}(A)$ is a semi-invariant differential.

Proof. The first and last statements are part of 2.2. The second statement is true, by 2.4 , if $A$ is a jacobian variety. There remains to be proved the second statement for an arbitrary $A$. Let then $A$ be arbitrary, and $k$ be of characteristic 0 . It is known that $A$ is the homomorphic image of a jacobian

(4) The result which we have in mind is a slight modification of the theorem on the extension of isomorphisms in a normal simple algebra; as this modification does not seem to be explicitly stated anywhere, we state and prove it in the Appendix at the end of this paper. 
variety; hence $A$ is isogenous to an abelian subvariety of a jacobian variety; since the $k$-modules of the closed differentials of the second kind, modulo exact differentials, of isogenous abelian varieties are isomorphic, we may assume $A$ to be a subvariety of the (nonsingular) jacobian $J$ of a curve $C$ without singular points. Let $g=\operatorname{dim} J$ be the genus of $C$. Let $J_{1}$ be a copy of $J$, and let $A_{1}$ be the copy of $A$ which is a subvariety of $J_{1}$; let $\lambda$ be a homomorphism of $J$ onto $A_{1}$, and let $\phi$ be a canonical mapping of $C$ into $J$. Then $\lambda \phi$ is a rational mapping of $C$ into $A_{1}$, also into $J_{1}$. Let $C_{1}$ be a copy of $C$, and let $\phi_{1}$ be the copy of $\phi$ which maps $C_{1}$ into $J_{1}$. According to Corollary 2 of Theorem 22 of [9], $\lambda$ is related to a class of algebraic correspondences between $C$ and $C_{1}$; let $D$ be an element of this class, so that $D$ is a virtual cycle on $C \times C_{1}$. For any $P \in C$, we have $\lambda \phi[P]=\pi_{i} e_{i} \phi_{1}\left[Q_{i}\right]$, if $\sum_{i} e_{i} Q_{\imath}=D\{P\}^{*}$; let $K$ be a finite extension of $k(C)$, such that $(D\{C\})_{K}$ is a sum of (not necessarily distinct) rational points $\Delta_{1}, \Delta_{2}, \cdots$ of $\left(C_{1}\right)_{K}$. Then $((\lambda \phi)\{C\})_{K}$ $=\prod_{i}\left(\phi_{1}\right)_{K}\left\{\Delta_{i}\right\}$. Let $B$ be a model of $K$ over $k$, without singularities, and let $\mu$ be the rational mapping of $B$ into $J_{1}$ such that $\mu\{B\}=((\lambda \phi)\{C\})_{K}$. Then the previous formula can be written $\mu=\sum_{i} \phi_{1} D_{i}$, where $D_{i}$ is the rational mapping of $B$ into $C_{1}$ such that $D_{i}\{B\}=\Delta_{i}$. Therefore, in the notation of the discussion preceding $2.5, \mu^{*}=\sum_{i}\left(\phi_{1} D_{i}\right)^{*}$; this means that for any $\omega \in \mathscr{D}_{2}(J)$, and for its copy $\omega_{1}$ in $D_{2}\left(J_{1}\right)$, we have $\mu^{*}\left(\omega_{1}\right)=\sum_{i}\left(\phi_{1}^{*}\left(\omega_{1}\right)\right)^{(i)}$, where $^{(i)}$ denotes extension on $B$ of a differential on $C_{1}$, when $k\left(C_{1}\right)$ is considered as a subfield of $K$ as prescribed by $\Delta_{i}$. Now, assume $D=\sum_{i} f_{i} H_{i}$, where the $H_{i}$ are distinct irreducible algebraic correspondences between $C$ and $C_{1}$; since $\mu^{*}\left(\omega_{1}\right)$ is the extension on $B$ of $(\lambda \phi)^{*}\left(\omega_{1}\right)$, the previous formula states that

$$
(\lambda \phi)^{*}\left(\omega_{1}\right)=\sum_{i} f_{i} T_{k\left(H_{i}\right) / k(C)}\left(\phi_{1}^{*}\left(\omega_{1}\right)\right)^{(i)},
$$

where: (1) for each $i, k(C)$ and $k\left(C_{1}\right)$ are considered as subfields of $k\left(H_{i}\right)$ as prescribed by $H_{i}$; (2) the symbol ( $)^{(i)}$ denotes extension on $H_{i}$ of a differential on $C$, or on $C_{1}$; (3) $T$ denotes trace.

Formula 2.9 can be simplified after introducing a different notation: we shall denote by $\mathscr{D}_{i}(D)$ (for $i=1,2, e$ ) the direct sum $\sum_{j} D_{i}\left(H_{j}\right)$; for $\Omega \in \mathscr{D}_{i}(C)$ (or $\mathscr{D}_{i}\left(C_{1}\right)$ ), $\Omega^{\prime}$ shall denote the element $\sum_{j} \Omega^{(j)}$ of $\mathscr{D}_{i}(D)$; for $\Omega \in \mathscr{D}_{i}(D)$, $T_{D / C} \Omega$ is defined as $\sum_{j} f_{j} T_{k\left(H_{j}\right) / k(C)} \Omega_{j}$, if $\Omega=\sum_{j} \Omega_{j}$ with $\Omega_{j} \in D_{i}\left(H_{j}\right)$; a similar definition holds for $T_{D / C_{1}} \Omega$. Finally, for $\Omega \in D_{i}(C)$ (or $D_{i}\left(C_{1}\right)$ ), $D^{*}(\Omega)$ is defined to be $T_{D / C_{1}} \Omega^{\prime}$ (or $T_{D / C} \Omega^{\prime}$ ). Then 2.9 becomes

\subsection{0}

$$
(\lambda \phi)^{*}\left(\omega_{1}\right)=D^{*}\left(\phi_{1}^{*}\left(\omega_{1}\right)\right) .
$$

This coincides with the correspondence, described in [7], between differentials of the first kind on curves related by an algebraic correspondence. If $\omega_{1}$ ranges in $D_{1}\left(J_{1}\right), \phi_{1}{ }^{*}\left(\omega_{1}\right)$ spans the whole $D_{1}\left(C_{1}\right)$, while

$$
(\lambda \phi)^{*}\left(\omega_{1}\right)=(\lambda \phi)^{*}\left(\omega_{1} \cap A_{1}\right) ;
$$


since $\omega_{1} \cap A_{1}$ ranges over the whole $D_{1}\left(A_{1}\right)$ by 2.5 , and since no proper abelian subvariety of $A_{1}$ contains $\lambda \phi C,(\lambda \phi)^{*}\left(\omega_{1}\right)$ spans a submodule of $D_{1}(C)$ of dimension $n$; thus, by 2.6 :

2.11. If $\Omega$ ranges in $D_{1}\left(C_{1}\right), D^{*}(\Omega)$ ranges over a submodule of $D_{1}(C)$ whose dimension $n$ is such that $2 n$ is the rank of the l-adic matrix (for an arbitrary prime l) related to the endomorphism of the jacobian of $C$ which represents the class of $D$.

Assume now $\omega_{1}$ to range over the set of the elements of $D_{2}\left(J_{1}\right)$ which are regular at $A_{1}$; let $m \leqq 2 g$ be the order of $D_{2}\left(A_{1}\right) / D_{e}\left(A_{1}\right)$. Then, again, $\phi_{1}^{*}\left(\omega_{1}\right)$ ranges over the whole $D_{2}\left(C_{1}\right)$, and $(\lambda \phi)^{*}\left(\omega_{1}\right)$ ranges over a submodule $Z$ of $D_{2}(C)$ such that $Z / Z \cap D_{e}(C)$ has order $m$. Thus, $Z$ is the $k$-module of the $D^{*}(\Omega)$ when $\Omega$ ranges over $D_{2}\left(C_{1}\right)$, and we have $m=$ ord $Z / Z \cap D_{e}(C)$. Let $V$ be the $k$-module of the $\Omega^{\prime}$ when $\Omega$ ranges in $D_{2}\left(C_{1}\right)$, and let $W$ be the $k$-module of the $\Omega \in D_{2}(D)$ such that $T_{D / C} \Omega \in D_{e}(C)$. Then, obviously,

$$
m=\operatorname{ord} Z / Z \cap D_{e(C)}=\text { ord } V / V \cap D_{e}(D)
$$

$$
-\operatorname{ord} V \cap W / V \cap D_{e}(D)=2 g-\operatorname{ord} V \cap W / V \cap D_{e}(D) .
$$

Now, let $Z^{\prime}, V^{\prime}, W^{\prime}$ be defined in the same manner as $Z, V, W$ respectively, after interchanging $C$ with $C_{1}$, and set $Z_{0}=Z^{\prime} \cap D_{1}\left(C_{1}\right), \quad V_{0}=V^{\prime} \cap D_{1}(D)$, $W_{0}=W^{\prime} \cap D_{1}(D)=$ set of all $\Omega \in D_{1}(D)$ such that $T_{D / C_{1}} \Omega=0$. Let $\lambda^{\prime}$ be the correspondent of $\lambda$ in the Rosati involution on $J$. The set of all $D^{*}(\Omega)$, when $\Omega$ ranges in $D_{1}(C)$, is $Z_{0}$; accordingly, by $2.11,2.7$ and $2.6, Z_{0}$ has order $n$; on the other hand, ord $Z_{0}=$ ord $V_{0}-$ ord $V_{0} \cap W_{0}$, so that

$$
\text { ord } V_{0} \cap W_{0}=\text { ord } V_{0}-n=g-n \text {. }
$$

If $\Omega \in \mathscr{D}_{1}(D)$ and $\Psi \in \mathscr{D}_{2}(D)$, and if $\Omega=\sum_{i} \Omega_{i}, \Psi=\sum_{i} \Psi_{i}$, with $\Omega_{i} \in D_{1}\left(H_{i}\right)$, $\Psi_{i} \in D_{2}\left(H_{i}\right)$, we shall define $j(\Psi, \Omega)$ to be $\sum_{i} f_{i} j\left(\Psi_{i}, \Omega_{i}\right)$. Hence, if $\Omega \in D_{1}(D)$ is such that $j(\Psi, \Omega)=0$ for each $\Psi \in V$, we also have $j\left(T_{D / C_{1}} \Psi, T_{D / C} \Omega\right)=0$ for each $\Psi \in V$, or $j\left(\Psi, T_{D / C_{1}} \Omega\right)=0$ for each $\Psi \in D_{1}\left(C_{1}\right)$; therefore, $T_{D / C_{1}} \Omega=0$, or $\Omega \in W_{0}$. Conversely, for $\Omega \in D_{1}(D), \Psi \in V$, and for any $\Omega^{*}$ conjugate to $\Omega$ over $k\left(C_{1}\right)$ (that is, if $\Omega=\sum_{i} \Omega_{i}, \Omega_{i} \in D_{1}\left(H_{i}\right)$, then $\Omega^{*}=\sum_{i} \Omega_{i}{ }^{*}$, with $\Omega_{i}{ }^{*}$ a conjugate of $\Omega_{i}$ on $k\left(C_{1}\right)$ ), we have $j(\Psi, \Omega)=j\left(\Psi, \Omega^{*}\right)$; hence $j(\Psi, \Omega)=0$ if $\Omega \in W_{0}$. This proves that $W_{0}$ is the set of all the $\Omega \in D_{1}(D)$ such that $j(\Psi, \Omega)=0$ for each $\Psi \in V$. In like manner we can prove that $W+\mathscr{D}_{1}(D)$ is the set of the $\Psi \in \mathscr{D}_{2}(D)$ such that $j(\Psi, \Omega)=0$ for each $\Omega \in V_{0}$; consequently, $V_{0}$ is the set of the $\Omega \in D_{1}(D)$ such that $j(\Psi, \Omega)=0$ for each $\Psi \in W$.

If $g_{i}$ is the genus of $H_{i}$, set $\gamma=\sum_{i} g_{i}$; then, in view of the duality between $D_{1}(D)$ and $D_{2}(D) / D_{1}(D)+D_{e}(D)$, we have proved that

ord $V \cap W / V \cap W \cap\left[D_{1}(D)+D_{e}(D)\right.$

$$
\begin{aligned}
& =\gamma-\operatorname{ord} V_{0}+W_{0}=\gamma-\left(\operatorname{ord} V_{0}+\operatorname{ord} W_{0}-\operatorname{ord} V_{0} \cap W_{0}\right) \\
& =\gamma-g-\operatorname{ord} W_{0}+\operatorname{ord} V_{0} \cap W_{0}=\gamma-n-\operatorname{ord} W_{0} \quad \text { by 2.13. }
\end{aligned}
$$


Now, the kernel of the homomorphic mapping $\Omega \rightarrow T_{D / C_{1}} \Omega$, of $D_{1}(D)$ onto $D_{1}\left(C_{1}\right)$, is $W_{0}$, so that ord $W_{0}=\gamma-g$, and

2.14

$$
\text { ord } V \cap W / V \cap W \cap\left[D_{1}(D)+D_{e}(D)\right]=g-n \text {. }
$$

But $W \cap\left[D_{1}(D)+D_{e}(D)\right]=\left[W \cap D_{1}(D)\right]+D_{e}(D)$, so that

ord $V \cap W / V \cap W \cap\left[D_{1}(D)+\mathscr{D}_{e}(D)\right]$

$$
\begin{aligned}
& =\operatorname{ord} V \cap W / V \cap\left[\left(W \cap D_{1}(D)\right)+D_{e}(D)\right] \\
& =\operatorname{ord}\left[V \cap W / V \cap D_{\epsilon}(D)\right] /\left[V \cap\left(\left(W \cap D_{1}(D)\right)+D_{e}(D)\right) / V \cap D_{e}(D)\right] \\
& =\text { ord } V \cap W / V \cap D_{e}(D)-\text { ord } V \cap W \cap D_{1}(D) ;
\end{aligned}
$$

the last term, by the formula analogous to 2.13 , has the value $g-n$, while the first member, by 2.14 , has the value $g-n$; hence ord $V \cap W / V \cap D_{e}(D)$ $=2(g-n)$. This, replaced in 2.12 , gives $m=2 g-2(g-n)=2 n$, Q.E.D.

3. Factor sets. The definitions of factor sets, and of constant factor sets, are given in $\$ \S 3$ and 4 of [4].

3.1. Theorem. Let $A$ be a nonsingular $n$-dimensional abelian variety over the algebraically closed field $k$ of characteristic zero; let $V$ be a 1-dimensional vector variety over $k$; denote by $\Gamma, \Gamma_{0}, \Gamma_{c}$ the groups of, respectively, the factor sets of $A$ into $V$, the factor sets associate to the identity of $\Gamma$, and the constant factor sets in $\Gamma$. Denote by $D_{2}^{\prime}, D_{e}^{\prime}$ the k-modules of the elements of, respectively, $\mathfrak{D}_{2}(A), \mathfrak{D}_{e}(A)$ which are regular at the identity of $A$. Then $\Gamma / \Gamma_{c}$ is isomorphic to $D_{2}^{\prime} / D_{1}(A)$, and in this isomorphism $\Gamma_{0} / \Gamma_{c}$ corresponds to $D_{e}^{\prime}$.

Proof. Let $A_{1}, A_{2}$ be copies of $A$, and let $v$ be a n.h.g.p. of $V$ such that the law of composition on $V$ is given by $v_{3}=v_{1}+v_{2}$. Denote by $\infty$ the point of $V$ at infinity for $v$. It is readily seen that the multiplicative notation for the law of composition on $V$ can be extended to yield $P \infty=\infty$ if $P \in V-\infty$. The associativity and commutativity properties remain true when meaningful. If $\gamma \in \Gamma-\Gamma_{c}$, assume $k(V) \subseteq k\left(A_{1} \times A_{2}\right)$ as prescribed by $\gamma$. Let $A_{3}$ be another copy of $A$, and assume $k\left(A_{3}\right) \subseteq k\left(A_{1} \times A_{2}\right)$ as prescribed by the law of composition on $A$. Let $W$ be the radical of the "denominator" of $v$ on $A_{1} \times A_{2}$; then $W=\gamma[\infty]$. The relation

$$
\left(\gamma\left[P_{1}+Q_{2} R_{2}\right]\right)\left(\gamma\left[Q_{1} \times R_{2}\right]\right)=\left(\gamma\left[P_{1} Q_{1} \times R_{2}\right]\right)\left(\gamma\left[P_{1} \times Q_{2}\right]\right),
$$

which characterizes factor sets, is now valid when: (a) none of the point. $P_{1} \times Q_{2} R_{2}, Q_{1} \times R_{2}, P_{1} Q_{1} \times R_{2}, P_{1} \times Q_{2}$ belongs to the fundamental locus $C$ of $?$ on $A_{1} \times A_{2}$, and (b) one factor at least on each side is not $\infty$. Let $H$ be a component of $W$, operating on the whole $A_{1} \times A_{2}$; we contend that there is a component of $W$ of the type $Y_{1} \times A_{2}$, where $Y$ is an $(n-1)$-dimensional irreducible subvariety of $A$, such that $H=T_{Y}^{\prime}$ (see $\S 4$ of [4] for the definition of $T_{Y}^{\prime}$ ). For if it were not so, it would be possible to find points $P, Q, R$ of $A$ such that $P_{1} \times Q_{2} \in H-(C \cap H)$, while $P_{1} Q_{1} \times R_{2}, P_{1} \times Q_{2} R_{2}$ and $Q_{1} \times R_{2}$ do not be- 
long to $W$; and this would contradict the previous formula. Conversely, let $Y$ be an $(n-1)$-dimensional irreducible subvariety of $A$ such that $Y_{1} \times A_{2}$ is a component of $W$, but assume $T_{Y}^{\prime}$ not to be a component of $W$. Then again it is possible to find points $P, Q, R$ of $A$ such that $P_{1} Q_{1} \times R_{2} \in W-C$, while $P_{1} \times Q_{2}, P_{1} \times Q_{2} R_{2}, Q_{1} \times R_{2}$ do not belong to $W$. Hence, since $\gamma\left[P_{1} \times Q_{2}\right]$ is symmetrical in $P, Q$, we conclude that there are distinct irreducible $(n-1)$ dimensional subvarieties $W^{1}, \cdots, W^{r}$ of $A$, none of which contains $E_{A}$, such that $W$ is the join of all the $W_{1}^{i} \times A_{2}, A_{1} \times W_{2}^{i}, T_{W^{i}}^{\prime}$.

If $P \in A$, let $\sigma_{P_{1}}, \sigma_{P_{2}}$ be the automorphisms of $k\left(A_{1} \times A_{2}\right)$ over, respectively, $k\left(A_{2}\right)$ and $k\left(A_{1}\right)$, which induce $\left(\sigma_{P}\right)_{1},\left(\sigma_{P}\right)_{2}$ in $k\left(A_{1}\right), k\left(A_{2}\right)$ respectively. If $P, Q \in A$, denote by $v\left(P_{1}, Q_{2}\right)$ the element of $k$ to which $v$ is congruent $\bmod \mathfrak{P}\left(P_{1} \times Q_{2} / A_{1} \times A_{2}\right)$, if $v \in Q\left(P_{1} \times Q_{2} / A_{1} \times A_{2}\right)$. Denote also by $v\left(P_{1}\right)$, $v\left(P_{2}\right)$ the elements of, respectively, $k\left(A_{2}\right), k\left(A_{1}\right)$ to which $v$ is congruent modulo, respectively, $\mathfrak{P}\left(P_{1} \times A_{2} / A_{1} \times A_{2}\right), \mathfrak{B}\left(A_{1} \times P_{2} / A_{1} \times A_{2}\right)$. Then formula 3.2 can be written

$$
\sigma_{Q_{2}}^{-1} v+v\left(Q_{1}\right)=\sigma_{Q_{1} v}^{-1}+v\left(Q_{2}\right),
$$

valid for $Q \notin W^{1} \cup \ldots \cup W^{r}$.

It has been proved in $\$ 7$ of [4] that, as a consequence of 3.3 , there exists a closed differential $\omega$ on $A$ such that

$$
d_{A_{1} \times A_{2}} v=\omega_{3}^{\prime}-\omega_{1}^{\prime}-\omega_{2}^{\prime},
$$

where $\omega_{i}^{\prime}$ is the extension on $A_{1} \times A_{2}$ of the copy $\omega_{i}$ of $\omega$ on $A_{i}$. This tells that $\omega$ is semi-invariant, so that $\omega \in D_{2}^{\prime}$ by 2.8 . The $\omega$ which satisfies 3.4 is unique but for an additive invariant differential; by 2.1 , this means that 3.4 establishes a homomorphism $\gamma \rightarrow \omega+D_{1}(A)$ of $\Gamma$ into $D_{2}^{\prime} / D_{1}(A)$, if $\omega$ is taken in $D_{1}(A)$ when $\gamma \in \Gamma_{c}$. It is also clear that $\gamma, \gamma^{\prime}$ correspond to the same $\omega+D_{1}(A)$ if and only if the difference of the corresponding $v, v^{\prime}$ has a differential $=0$, that is, if and only if $\gamma-\gamma^{\prime} \in \Gamma_{c}$; hence 3.4 establishes an isomorphic mapping of $\Gamma / \Gamma_{c}$ into $D_{2}^{\prime} / D_{1}(A)$. Finally, $\gamma \in \Gamma_{0}$ if and only if $\omega$ can be selected in $D_{e}^{\prime}$.

We must now prove that this isomorphic mapping is onto $D_{2}^{\prime} / D_{1}(A)$; given $\omega \in D_{2}^{\prime}$, we shall select any $\gamma \in \Gamma_{c}$ if $\omega \in D_{1}(A)$; if not, let $\{x\},\{y\},\{z\}$ be n.h.g.p. of $A_{1}, A_{2}, A_{3}$ respectively, copies of each other, and let $X$ be the rational point of $\left(A_{2}\right)_{k\left(A_{1}\right)}$ at which $y_{i}=x_{i}$. By 2.8 , we have $\left(\omega_{2}\right)_{k\left(A_{1}\right)}$ $\sim \sigma_{\bar{X}}^{-1}\left(\omega_{2}\right)_{k\left(A_{1}\right)}$; let $\left\{v_{i}^{1}, \cdots, v_{i}^{n}\right\}(i=1,2,3)$ be $k$-bases for $D_{1}\left(A_{i}\right)$, copies of each other, and write $\omega_{i}=\sum_{j} a_{i}^{j} v_{i}^{j}$, with $a_{i}^{j} \in k\left(A_{i}\right)$. Then there exists a $t \in k\left(A_{1} \times A_{2}\right)$ such that $\sum_{j} a_{2}^{j}\left(v_{2}^{j}\right)_{k\left(A_{1}\right)}=\sum_{j} a_{3}^{j}\left(v_{2}^{j}\right)_{k\left(A_{1}\right)}-\sum_{j}\left(D_{2}^{j} t\right)\left(v_{2}^{j}\right)_{k\left(A_{1}\right)}$, where $\left\{D_{2}^{j}\right\}$ is the $k$-basis for the invariant derivations on $\left(A_{2}\right)_{k\left(A_{1}\right)}$ such that $D_{2}^{j}\left(v_{2}^{i}\right)_{k\left(A_{1}\right)}=\delta_{i j}$. Set $v_{0}^{j}=\left(\right.$ extension of $v_{3}^{j}$ on $\left.A_{1} \times A_{2}\right)=v_{1}^{j} \times A_{2}+v_{2}^{j} \times A_{1}$; then the previous formula can be written:

$$
\sum_{j} a_{2}^{j j} v_{2}^{j} \times A_{1}=\sum_{j} a_{3}^{j} v_{0}^{j}-d_{A_{1} \times A_{2}} t-\sum_{j} b_{j} v_{1}^{j} \times A_{2},
$$


for suitable $b_{j} \in k\left(A_{1} \times A_{2}\right)$. This, in turn, gives, if $D_{1}^{j}$ are the copies of $D_{2}^{j}$ on $\left(A_{1}\right)_{k\left(A_{2}\right)}$ :

$$
\sum_{j} b_{j}\left(v_{1}^{j}\right)_{k\left(A_{2}\right)}=\sum_{j} a_{3}^{j}\left(v_{1}^{j}\right)_{k\left(A_{2}\right)}-\sum_{j}\left(D_{1}^{j} t\right)\left(\begin{array}{c}
j \\
v_{1}
\end{array}\right)_{k\left(A_{2}\right)} ;
$$

this means that $\sum_{j} b_{j}\left(v_{1}^{j}\right)_{k\left(A_{2}\right)} \sim \sigma_{Y}^{-1}\left(\omega_{1}\right)_{k\left(A_{2}\right)}$, where $Y$ is the rational point of $\left(A_{1}\right)_{k\left(A_{2}\right)}$ at which $x_{i}=y_{i}$. Since $\sigma_{Y}^{-1}\left(\omega_{1}\right)_{k\left(A_{2}\right)} \sim\left(\omega_{1}\right)_{k\left(A_{2}\right)}$, it follows that $\sum_{j} b_{j}\left(v_{1}^{j}\right)_{k\left(A_{2}\right)} \sim\left(\omega_{1}\right)_{k\left(A_{2}\right)}$. Considering that the left side, and the first two terms of the right side of 3.5 , are closed differentials on $A_{1} \times A_{2}$, we see that the same is true of $\sum_{j} b_{j} v_{1}^{j} \times A_{2}$, so that $D_{2}^{i} b_{j}=0$ for each $i, j$, and therefore $b_{j} \in k\left(A_{1}\right)$. But then $\sum_{j} b_{j} v_{1}^{j} \sim \omega_{1}$, or $\sum_{j} b_{j} v_{1}^{j}=\omega_{1}+d_{A_{1}} t^{\prime}$, for $t^{\prime} \in k\left(A_{1}\right)$. If $u=t+t^{\prime}, 3.5$ now gives

$$
d_{A_{1} \times A_{2}} u=\omega_{3}^{\prime}-\omega_{1}^{\prime}-\omega_{2}^{\prime},
$$

which is analogous to 3.4. Let $\gamma$ be the rational mapping of $A_{1} \times A_{2}$ into $V$ generated by setting $v=u$; we contend that $\gamma$ is a factor set. If it is, it is clearly unique but for an additive constant set, since $u$ is determined but for an additive element of $k$.

Now, $\gamma\left[E_{A_{1}} \times E_{A_{2}}\right]$ is a point $\neq \infty$ of $V$, because $E_{A_{1}} \times E_{A_{2}}$ does not belong to a pole of $\omega_{3}^{\prime}-\omega_{1}^{\prime}-\omega_{2}^{\prime}$. Moreover, the operation of interchanging $A_{1}$ with $A_{2}$ transforms $u$ into $u+h$, with $h \in k$; application of the same operation again shows that $2 h=0$, or $h=0$. Hence $\gamma\left[P_{1} \times Q_{2}\right]=\gamma\left[Q_{1} \times P_{2}\right]$ for generic $P, Q \in A$.

For a generic $P \in A, 3.6$ implies that $d_{2} \sigma_{P_{1}}^{-1} u=\sigma_{P_{2}}^{-1} \omega_{3}^{\prime}-\omega_{2}^{\prime}$, where $d_{2}$ indicates construction of differential on $\left(A_{2}\right)_{k\left(A_{1}\right)}$; also, $d_{2} \sigma_{P_{2}}^{-1} u=\sigma_{P_{2}}^{-1} \omega_{3}^{\prime}-\sigma_{P_{2}}^{-1} \omega_{2}^{\prime}$; hence $\quad d_{2}\left(\sigma_{P_{1}}^{-1} u-\sigma_{P_{2}}^{-1} u\right)=\sigma_{P_{2}}^{-1} \omega_{2}^{\prime}-\omega_{2}^{\prime}=\left(\left(\sigma_{P}^{-1} \omega-\omega\right)_{2}\right)_{k\left(A_{1}\right)}$. But $\quad\left(\sigma_{P}^{-1} \omega-\omega\right)_{2}$ $=d_{A_{2}} u\left(P_{1}\right)$ by 3.6. Hence there exists a $w \in k\left(A_{1}\right)$ such that $w+\sigma_{P_{1}}^{-1} u$ $=\sigma_{P_{2}}^{-1} u+u\left(P_{1}\right)$. Since $P$ is generic, the elements $\left(\sigma_{P_{1}}^{-1} u\right)\left(E_{A_{2}}\right),\left(\sigma_{P_{2}}^{-1} u\right)\left(E_{A_{2}}\right)$, $\left(u\left(P_{1}\right)\right)\left(E_{A_{2}}\right)$ exist, and equal respectively $\left(\sigma_{P}^{-1}\right)_{1}\left(u\left(E_{A_{2}}\right)\right), u\left(P_{2}\right), u\left(P_{1}, E_{A_{2}}\right)$. But $u\left(E_{A_{2}}\right) \in k$, and therefore it coincides with $u\left(P_{1}, E_{A_{2}}\right)$. Hence $w=u\left(P_{2}\right)$, so that $\sigma_{P_{1}}^{-1} u+u\left(P_{2}\right)=\sigma_{P_{2}}^{-1} u+u\left(P_{1}\right)$. This is the same as 3.3 , and is therefore equivalent to 3.2 , Q.E.D.

From $3.1,2.8$, and the fact that, by $2.2, D_{2}^{\prime} / D_{1}(A)+D_{e}^{\prime} \cong D_{2}(A) / D_{1}(A)$ $+D_{e}(A)$, we obtain:

3.7. Corollary. Notations as in 3.1. Then $\Gamma / \Gamma_{0}$ is a k-module isomorphic to $\mathfrak{D}_{2}(A) / D_{1}(A)+\mathfrak{D}_{e}(A)$, and has order $n=\operatorname{dim} A$.

Appendix (Added November 15, 1955). In the proof of 2.7 (see footnote in that proof) we have made use of the following result:

THEOREM. Let $Q$ be a total matrix algebra over the field $F$; let $B, B^{\prime}$ be subrings of $Q$, such that $B \cap F=B^{\prime} \cap F$ is a perfect subfield $f$ of $F$, and assume $B$, $B^{\prime}$ to be simple algebras over $f$, isomorphic to each other in an isomorphism $\sigma$ over $f$. Assume also that for any element $y$ of the center of $B$, or of $\mathbb{B}^{\prime}$, the characteristic 
polynomial of the matrix $y$ has coefficients in $f$. Then there exists an inner automorphism of $a$ which induces $\sigma$ between $B$ and $B^{\prime}$.

Proof. Let $\mathfrak{e}, \mathfrak{e}^{\prime}$ be the centers of $B, B^{\prime}$ respectively; since $f$ is perfect, we have $\mathfrak{C}=f(\theta)$ for a suitable $\theta \in \mathcal{C}$, and $\mathbb{C}^{\prime}=f\left(\theta^{\prime}\right)$ if $\theta^{\prime}=\sigma \theta$. For an indeterminate $x$, let $g(x), g_{0}(x), c(x)$ be respectively the minimal polynomial of $\theta$ over $f$, the minimal polynomial of $\theta$ over $F$, and the characteristic polynomial of the matrix $\theta$; let $g^{\prime}(x)=g(x), g_{0}^{\prime}(x), c^{\prime}(x)$ have the same respective offices for $\theta^{\prime}$. Then $g_{0}$ is a divisor of $g, g$ is a divisor of $c$, and the zeros of $g_{0}$ (in some algebraic closure of $F$ ) are all and only the zeros of $c$; since $g$ is separable, and has therefore distinct zeros, this implies that $g=g_{0}$, and likewise $g=g_{0}^{\prime}$; also, $c=g^{r}$ for some positive integer $r$, and likewise $c^{\prime}=g^{r}$. Thus the matrices $\theta$ and $\theta^{\prime}$ have the same characteristic polynomial, and of course $F(\theta)$ and $F\left(\theta^{\prime}\right)$ are semisimple algebras. This suffices to show that there exists an inner automorphism $\rho$ of $a$ such that $\rho \theta=\theta^{\prime}$; thus, $\rho$ induces $\sigma$ between $\mathfrak{C}$ and $\mathfrak{C}^{\prime}$.

The smallest sub-algebra $F(\theta)$ of $Q$ which contains $\mathcal{C}$ and $F$ is a semi-field, that is, a direct sum of fields, say $\mathfrak{C}_{1} \dot{+} \ldots \dot{+} \mathfrak{C}_{s}$; likewise, the smallest subalgebra of $a$ which contains $\mathfrak{C}^{\prime}$ and $F$ is $\mathfrak{C}_{1}^{\prime} \dot{+} \cdots+\mathfrak{C}_{s}^{\prime}$, where $\mathfrak{C}_{i}^{\prime}=\rho \mathfrak{C}_{i}$; since $\theta$ has the same degree over $f$ or $F$, we have $\mathfrak{C}_{1} \dot{+} \cdots+\mathfrak{e}_{8}=\mathfrak{e}_{F}=\mathfrak{e} \times F$ (direct product over $f$ ). This proves that the smallest sub-algebra of $a$ which contains $B$ and $F$ is $B \times F=B_{F}$, and it is known that $B_{F}=B_{1} \dot{+} \cdots+B_{s}, B_{i}$ being a simple algebra over $F$, with the center $\mathfrak{C}_{i}$; similarly, the smallest subalgebra of $a$ which contains $B^{\prime}$ and $F$ is $B^{\prime} \times F=B_{F}^{\prime}=B_{1}^{\prime}+\cdots+B_{s}^{\prime}$. But then, $\sigma$ can be extended to an isomorphism of $B_{F}$ onto $B_{F}^{\prime}$ over $F$, and we have of course $\sigma B_{i}=B_{i}^{\prime}$. Let $e_{i}$ be the unit element of $\bigotimes_{i}$, and set $Q_{i}=e_{i} Q e_{i}$; then $Q_{i}$ is a total matrix algebra over $F$, containing, as simple sub-algebras, $B_{i}$ and $\rho^{-1} \sigma B_{i}$, since $\sigma B_{i}$ has the unit element $\sigma e_{i}=\rho e_{i}$, and is therefore a subalgebra of $\left(\rho e_{i}\right) Q\left(\rho e_{i}\right)=\rho Q_{i}$. Thus, by the theorem on the extension of isomorphisms between simple sub-algebras of a normal simple algebra, there is an inner automorphism

$$
\tau_{i}: y \rightarrow t_{i} y t_{i}^{-1}
$$

of $Q_{i}$ which induces $\rho^{-1} \sigma$ between $B_{i}$ and $\rho^{-1} \sigma \cap_{i}$. Set $t=t_{1}+\cdots+t_{s}$, and let $\tau$ be the inner automorphism $y \rightarrow t y t^{-1}$ of $\propto$. For a $y \in ß$, write $y=\sum_{i} y_{i}$, with $y_{i} \in \bigotimes_{i}$; then $\sigma y=\sum_{i} \sigma y_{i}=\sum_{i} \rho \tau_{i} y_{i}=\rho \tau y$, Q.E.D.

\section{BIBLIOGRAPHY}

1. I. Barsotti, Algebre senza base finita (II), Annali di Matematica Pura ed Applicata vol27 (1948) p. 243; see also Errata, ibid. vol. 28 (1949) p. 346.

2. - Local properties of algebraic correspondences, Trans. Amer. Math. Soc. vol. 71 (1951) p. 349.

3. —, A note on abelian varieties, Rend. Circ. Mat. Palermo vol. 2 (1953) p. 236.

4. - Structure theorems for group-varieties, Annali di Matematica Pura ed Applicata vol. 38 (1955) p. 77. 
5. - - Un teorema di struttura per le varietà gruppali, Rend. Acc. Naz. Lincei vol. 18 (1955) p. 43.

6. C. Chevalley, Introduction to the theory of algebraic functions of one variable, New York, 1951.

7. M. Deuring, Arithmetische theorie der korrespondenzen algebraischer funktionenkörper II, J. Reine Angew. Math. vol. 183 (1941) p. 25.

8. W. Krull, Dimensionstheorie in stellenringen, J. Reine Angew. Math. vol. 179 (1938) p. 204.

9. A. Weil, Variêtés abéliennes et courbes algébriques, Actualités Scientifiques et Industrielles, no. 1064, Paris, 1948.

University of Pittsburgh, Pittsburgh, PA.

University of Southern California, Los Angeles, Calif. 